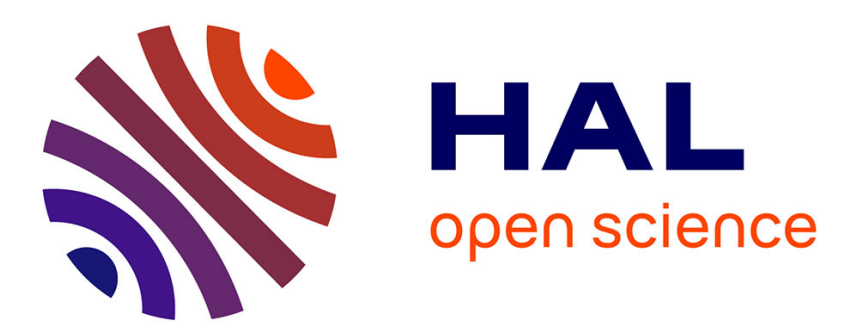

\title{
Charge localization and magnetic correlations in the refined structure of $\mathrm{U} 3 \mathrm{O} 7$
}

Gregory Leinders, Gianguido Baldinozzi, Clemens Ritter, Rolando Saniz, Ine Arts, Dirk Lamoen, Marc Verwerft

\section{- To cite this version:}

Gregory Leinders, Gianguido Baldinozzi, Clemens Ritter, Rolando Saniz, Ine Arts, et al.. Charge localization and magnetic correlations in the refined structure of U3O7. Inorganic Chemistry, In press, 10.1021/acs.inorgchem.1c01212 . hal-03271055

\section{HAL Id: hal-03271055 \\ https://hal.science/hal-03271055}

Submitted on 25 Jun 2021

HAL is a multi-disciplinary open access archive for the deposit and dissemination of scientific research documents, whether they are published or not. The documents may come from teaching and research institutions in France or abroad, or from public or private research centers.
L'archive ouverte pluridisciplinaire HAL, est destinée au dépôt et à la diffusion de documents scientifiques de niveau recherche, publiés ou non, émanant des établissements d'enseignement et de recherche français ou étrangers, des laboratoires publics ou privés. 


\section{Charge localization and magnetic correlations in the refined structure of $\mathrm{U}_{3} \mathrm{O}_{7}$}

\section{Gregory Leinders, ${ }^{\text {a, }}$ " Gianguido Baldinozzi, ${ }^{\mathrm{b}}$ Clemens Ritter, ${ }^{\mathrm{c}}$ Rolando Saniz, ${ }^{\text {d }}$ Ine Arts, ${ }^{\mathrm{e}}$ Dirk Lamoen, ${ }^{\mathrm{e}}$ Marc Verwerft ${ }^{\mathrm{a}}$}

a Belgian Nuclear Research Centre (SCK CEN), Institute for Nuclear Materials Science, Boeretang 200, B-2400 Mol, Belgium.

b Université Paris-Saclay, CentraleSupélec, CNRS, SPMS, 91190 Gif-sur-Yvette, France.

c Institut Laue-Langevin, 71 Avenue des Martyrs, 38042 Grenoble, France.

d CMT \& NanoLab Center of Excellence, Department of Physics, University of Antwerp, Groenenborgerlaan 171, B-2020 Antwerpen, Belgium.

e EMAT \& NanoLab Center of Excellence, Department of Physics, University of Antwerp, Groenenborgerlaan 171, B-2020 Antwerpen, Belgium.

\footnotetext{
* Corresponding author

E-mail address: gregory.leinders@sckcen.be (G. Leinders)

Phone: +3214333163
} 


\begin{abstract}
Atomic arrangements in the mixed valence oxide $\mathrm{U}_{3} \mathrm{O}_{7}$ are refined from high-resolution neutron scattering data. The crystallographic model describes long-range structural order in a $\mathrm{U}_{60} \mathrm{O}_{140}$ primitive cell (space group $\mathrm{P}_{2} / n$ ) containing distorted cuboctahedral oxygen clusters. By combining experimental data and electronic structure calculations accounting for spinorbit interactions, we provide robust evidence of an interplay between charge localization and the magnetic moments carried by the uranium atoms. The calculations predict $\mathrm{U}_{3} \mathrm{O}_{7}$ to be a semiconducting solid with a band gap of close to $0.32 \mathrm{eV}$, and a more pronounced chargetransfer insulator behavior as compared to the well-known Mott insulator $\mathrm{UO}_{2}$. Most uranium ions (56 out of 60) occur in nine-fold and ten-fold coordinated environments, surrounding the oxygen clusters, and have a tetravalent ( 24 out of 60 ) or pentavalent ( 32 out of 60 ) state. The remaining uranium ions ( 4 out of 60 ) are not contiguous to the oxygen cuboctahedra, and have a very compact, eight-fold coordinated environment with two short $(2 \times 1.93(3) \AA)$ "oxo-type" bonds. The higher Hirshfeld charge and the diamagnetic character point to a hexavalent state for these four uranium ions. Hence, the valence state distribution corresponds to $24 / 60 \times \mathrm{U}(\mathrm{IV})+32 / 60 \mathrm{U}(\mathrm{V})+4 / 60 \mathrm{U}(\mathrm{VI})$. The tetravalent and pentavalent uranium ions are predicted to carry non-collinear magnetic moments (with amplitudes of $1.6 \mu_{\mathrm{B}}$ and $0.8 \mu_{\mathrm{B}}$, respectively), resulting in canted ferromagnetic order in characteristic layers within the overall fluorite-related structure.
\end{abstract}




\section{Introduction}

Due to its wide range of oxidation states, the coordination chemistry of uranium is today rapidly expanding and uranium complexes are being studied in many application fields, reaching beyond that of its more common use as nuclear fuel. ${ }^{1,2}$ Nevertheless, despite decades of ongoing research, even the binary $\mathrm{U}-\mathrm{O}$ system continues to challenge researchers and engineers. ${ }^{3,4}$ Uranium(IV)oxide $\left(\mathrm{UO}_{2}\right)$ remains the dominant component for today's fuels used by commercial nuclear power reactors. Many of its physical properties such as density, thermal conductivity, self-diffusion, impurity and fission product diffusion are all highly dependent of slight deviations from exact stoichiometry. ${ }^{5}$

A significant body of work has been published on the thermodynamics of the $\mathrm{U}-\mathrm{O}$ binary system as well as on the oxidation kinetics of $\mathrm{UO}_{2}$ under dry and wet conditions. ${ }^{6-10}$ Oxidation alters the original fluorite-type crystal lattice by forming oxygen defects and defect clusters. ${ }^{11}$ At temperatures above $700 \mathrm{~K}$, a wide non-stoichiometric solid solution phase field $\left(\mathrm{UO}_{2+x}\right)$ gradually develops, but at lower temperatures only slight deviations from stoichiometry $(0<x<0.03)$ can occur. ${ }^{9}$ Upon further oxidation in the low-temperature region, morphotropic phase transformations take place with formation of mixed-valence compounds, commonly referred to as $\mathrm{U}_{4} \mathrm{O}_{9}, \mathrm{U}_{3} \mathrm{O}_{7}$, and $\mathrm{U}_{3} \mathrm{O}_{8} .{ }^{12-15}$ The oxides $\mathrm{U}_{4} \mathrm{O}_{9}$ and $\mathrm{U}_{3} \mathrm{O}_{7}$ are structurally closely related to the original fluorite-type crystal lattice, while the transformation to $\mathrm{U}_{3} \mathrm{O}_{8}$ induces a more drastic crystallographic reorganization which is associated with a considerable volume increase (36 vol.\%).

Uranium oxides are recognized as a small-gap semiconductor (with either Mott or charge transfer insulator characteristics) where strong interactions exist at low temperature between the electronic and lattice degrees of freedom. ${ }^{16}$ The magnetic structure of $\mathrm{UO}_{2}$ has been studied extensively during the past 60 years, ${ }^{17,18}$ though certain details are not fully understood yet. The situation is even less established for the higher oxides retaining a fluoritetype structure such as $\mathrm{U}_{4} \mathrm{O}_{9}$ and $\mathrm{U}_{3} \mathrm{O}_{7}$ where some of the tetravalent $\mathrm{U}(\mathrm{IV})$ uranium ions are replaced by pentavalent $\mathrm{U}(\mathrm{V})$ or hexavalent $\mathrm{U}(\mathrm{VI})$ ions. Nevertheless, magnetic properties have been characterized in a multitude of uranium complexes. ${ }^{19,20}$

Numerous first-principles studies of binary uranium oxides have been published in recent years. Most of them within the Density Functional Theory (DFT) approach, thereby combining the Perdew-Burke-Ernzerhof functional and an on-site Coulomb repulsion between localized $f$ electrons ( $\mathrm{PBE}+U),{ }^{21,22}$ using the parametrization of Dudarev and co-workers in their well-known study of $\mathrm{UO}_{2} .{ }^{23}$ In addition to further studies on $\mathrm{UO}_{2},{ }^{24-27}$ also $\mathrm{U}_{4} \mathrm{O}_{9},{ }^{25,26,28}$ $\mathrm{U}_{3} \mathrm{O}_{7},{ }^{25,26,28}$ and $\mathrm{U}_{3} \mathrm{O}_{8}$ were investigated extensively. ${ }^{25,26,28-30}$ Point defects in $\mathrm{UO}_{2}$ have also been studied following the $\mathrm{PBE}+U$ approach, ${ }^{31}$ as well as oxygen cluster stability in $\mathrm{UO}_{2+x} .{ }^{32,33}$ The Heyd-Scuseria-Ernzerhof (HSE) hybrid functional approach, ${ }^{34}$ which is computationally more expensive, has been less applied to study the uranium oxides so far, and 
only to address smaller unit cell systems, such as $\mathrm{UO}_{2}$ or $\mathrm{U}_{3} \mathrm{O}_{8} \cdot{ }^{25,27,29,35}$ We note that spinorbit coupling (SOC) is often neglected, although uranium is a heavy element. The main reason for neglecting this correction is the reduction in computational cost. Researchers that do take it into account, however, have reported dissimilar conclusions. While earlier studies on actinide dioxides, including $\mathrm{UO}_{2}$, found that the effect of SOC is subsidiary, ${ }^{29,36}$ more recent studies on $\mathrm{UO}_{2}$ find that it favors the correct $3 \mathrm{k}$ magnetic order in its ground state. ${ }^{18,27}$ In the progressive oxidation of the $\mathrm{U}-\mathrm{O}$ system, $\mathrm{U}_{3} \mathrm{O}_{7}$ is the last compound with a fluoriterelated structure before the transformation to the layered structure of $\mathrm{U}_{3} \mathrm{O}_{8}$, which makes it an interesting structure to unravel. Contrary to the parent compounds $\mathrm{UO}_{2}$ and $\mathrm{U}_{4} \mathrm{O}_{9}$, the $\mathrm{U}_{3} \mathrm{O}_{7}$ phase is characterized by a tetragonal distortion of the formerly cubic lattice. Various experiments showed a gradually increasing axial ratio of $1.000<c / a<1.031,{ }^{12,14,37,38}$ indicative of a sub-stoichiometric phase field $\mathrm{U}_{3} \mathrm{O}_{7-z}$. Additionally, a lattice strain effect attributed to topotactic growth of $\mathrm{U}_{3} \mathrm{O}_{7}$ onto $\mathrm{U}_{4} \mathrm{O}_{9}$ has been considered to affect the stoichiometry and axial ratio of the $\mathrm{U}_{3} \mathrm{O}_{7}$ phase. ${ }^{13}$ At the oxygen-rich boundary, however, the oxygen-to-uranium ratio $(\mathrm{O} / \mathrm{U})$ was determined as $2.34 \pm 0.01$, consistent with the nominal composition $\mathrm{U}_{3} \mathrm{O}_{7}(\mathrm{O} / \mathrm{U}=2.333){ }^{39}$

Until recently, the crystal symmetry of phase-pure $\mathrm{U}_{3} \mathrm{O}_{7}$ remained inconclusive and atomic arrangements unconfirmed, despite ongoing theoretical and experimental efforts. ${ }^{28,40-42}$ Electron diffraction investigations have confirmed the (pseudo-cubic) tetragonal distortion of the fluorite-type parent structure, and additionally revealed a long-range ordered superstructure, characterized by sharp satellite reflections. ${ }^{39}$ Similar observations exist for the related system of $\mathrm{U}_{4} \mathrm{O}_{9},{ }^{43}$ but the symmetries of the long-range order are substantially different from each other. The superstructure of $\mathrm{U}_{3} \mathrm{O}_{7}$ proved to be commensurate with the parent fluorite unit cell, but with a non-trivial orientation relationship between the fluorite lattice vectors and the lattice vectors of the defect ordering. It is described in an expanded cell with a $P 4_{2} / n$ space group symmetry, comprising fifteen fluorite cells. By combining the symmetry of the defect ordering in $\mathrm{U}_{3} \mathrm{O}_{7}$ with the cuboctahedral anion cluster model proposed originally by Bevan et al. for anion-excess fluorites, ${ }^{44,45}$ a self-consistent trial model was proposed..$^{39}$ In this trial model the structural symmetry derived from the diffraction experiments was maintained, and the unit cell composition (i.e. $\mathrm{U}_{60} \mathrm{O}_{140}$ ) satisfied the nominal $\mathrm{O} / \mathrm{U}$ ratio of 2.333. Importantly, bond valence sum analysis and an in-depth assessment of the electron diffraction data indicated that the geometry of the cuboctahedral cluster model would need to be distorted, but the data did not enable further refinements of the atomic positions.

The charge localization mechanism in mixed-valence uranium oxides is a fundamental aspect of interest to a broad scientific community. In the fluorite-derived compounds $\mathrm{U}_{4} \mathrm{O}_{9}$ and $\mathrm{U}_{3} \mathrm{O}_{7}$ mainly the $\mathrm{U}(\mathrm{IV})$ and $\mathrm{U}(\mathrm{V})$ oxidation states occur for the uranium ions, whereas $\mathrm{U}_{3} \mathrm{O}_{8}$ contains a mixture of $\mathrm{U}(\mathrm{V})$ and $\mathrm{U}(\mathrm{VI})$ species. ${ }^{38,46,47}$ Interestingly, a minority fraction of $\mathrm{U}(\mathrm{VI})$ has also been reported for the fluorite-derived compounds. ${ }^{48,49}$ The associated uranium 
coordination chemistry is, however, not straightforward. In addition to simple cubic and octahedral coordination environments also pentagonal bipyramids, square antiprisms and various distortions of the former polyhedra can be identified. Evidently, the uranium speciation is related to the type of oxygen clusters present in these oxide phases. Recent explorations of the $\mathrm{U}-\mathrm{O}$ radial distribution in the $\mathrm{U}_{3} \mathrm{O}_{7}$ phase from Extended X-ray Absorption Fine Structure (EXAFS) data confirmed the cuboctahedral oxygen cluster as a suitable building block in the structural model, but further indicated that its local deformation is likely different from the trial model proposed before. ${ }^{38}$

In this work, we present the results of X-ray experiments performed at room temperature and neutron diffraction experiments conducted at $1.5 \mathrm{~K}, 100 \mathrm{~K}$ and $298 \mathrm{~K}$ on a polycrystalline $\mathrm{U}_{3} \mathrm{O}_{7}$ powder sample prepared at thermal equilibrium. The aim was to accurately refine atomic arrangements, and to determine the electronic structure by spin-resolved densityfunctional theory calculations. The corroborating experimental and calculated results allowed to derive a robust model of the charge localization in $\mathrm{U}_{3} \mathrm{O}_{7}$. Additionally, the occurrence of long-range magnetic order was evaluated from the neutron diffraction data, and predictions within the electronic structure calculations. 


\section{Materials and methods}

\subsection{Sample preparation}

Polycrystalline $\mathrm{U}_{3} \mathrm{O}_{7}$ powder was prepared by performing a controlled oxidation of depleted, nuclear grade $\mathrm{UO}_{2+x}$ powder supplied by FBFC International (Dessel, Belgium).

Approximately $5 \mathrm{~g}$ of the feed material was heated to $523 \mathrm{~K}$ at a rate of $5 \mathrm{~K} \mathrm{~min}^{-1}$ in a Nabertherm LT 9113/P330 muffle furnace, under normal atmospheric conditions. An isotherm duration of $7 \mathrm{~h}$ was applied before cooling back to room temperature $\left(10 \mathrm{~K} \mathrm{~min}^{-1}\right)$. The stoichiometry $(\mathrm{O} / \mathrm{U})$ was measured by gravimetric analysis and was equal to $2.333 \pm 0.001$, and additionally, powder X-ray diffraction was performed to confirm the phase purity.

\subsection{Diffraction techniques}

Room temperature X-ray diffraction (XRD) was performed on a Bruker D8 Advance diffractometer, in a vertical Bragg-Brentano geometry. The radiation source consisted of an

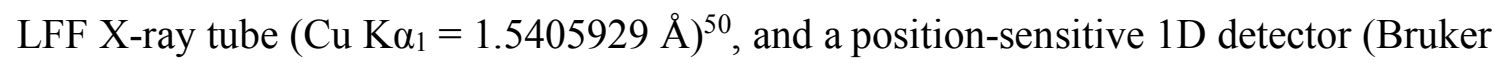
Lynxeye) with an active length of $3.3^{\circ} 2 \theta$ was used. Goniometer alignment validation was performed on a weekly basis, using a standard reference material (NIST SRM1976b). The $\mathrm{U}_{3} \mathrm{O}_{7}$ sample was prepared in a designated sample holder via the standard back-loading technique for polycrystalline powders.

The incident beam optics consisted of a fixed divergence slit $\left(1 / 2^{\circ}\right)$ and a copper beam mask to control the spot size. Soller slit assemblies $\left(1.6^{\circ}\right)$ were inserted in both incident and diffracted beam paths to limit axial divergence. The sample holder was placed on a rotation stage set to 15 rotations per minute. A beam knife was positioned above the sample to reduce scatter at low incident angles. The $\mathrm{Cu} \mathrm{K} \beta$ contribution to the diffracted signal was largely removed by application of a Ni filter in front of the detector. The pattern was measured through the range $20-122^{\circ} 2 \theta$, using a step size of $0.018^{\circ}$.

Neutron diffraction data was collected at the D2B and D20 beamlines of the Institut LaueLangevin (France). ${ }^{51,52}$ At D2B the data were acquired with a wavelength of $1.594 \AA$ at $1.5 \mathrm{~K}$ and at room temperature $(298 \mathrm{~K})$, using a standard orange cryostat. At D20 the wavelength $2.41 \AA$ was selected using the high flux $42^{\circ}$ takeoff angle. Measurements were performed at $1.5 \mathrm{~K}$ and at $100 \mathrm{~K}$, also using a standard orange cryostat. The sample consisted of $\mathrm{U}_{3} \mathrm{O}_{7}$ polycrystalline powder, loaded into a cylindrical vanadium canister (6 $\mathrm{mm}$ diameter). The same sample was used for the acquisitions at both beamlines.

Data evaluation and full-pattern refinement (Rietveld method) were performed with the JANA2006 software package. ${ }^{53}$ A systematic approach was followed, with consecutive refinement operations on a parameter set which included the scale factor, a $5^{\text {th }}$ order Cheybchev background function, lattice parameters, sample displacement or zero-shift, and a pseudo-Voigt profile function. Specific details are given in the corresponding subsections of 
this work when isotropic thermal displacement parameters and atomic positions were additionally refined. Each refinement cycle was executed until the ratio of numeric shift $(\delta p)$ to estimated standard deviation $(\delta s)$ for each checked parameter was smaller than 0.05 . The convergence was also evaluated from the expected and weighted-profile $R$-factors $\left(R_{\exp }\right.$ and $R_{\mathrm{wp}}$, respectively), and the goodness-of-fit value $(\chi)$ :

$R_{\exp }=\sqrt{\frac{\#(\text { profile points })-\#(\text { variables })}{\sum w_{i} \cdot y_{i}(\mathrm{obs})^{2}}} \cdot 100$,

$R_{\mathrm{wp}}=\sqrt{\frac{\sum w_{i} \cdot\left(y_{i}(\mathrm{obs})-y_{i}(\mathrm{calc})\right)^{2}}{\sum w_{i} \cdot y_{i}(\mathrm{obs})^{2}}} \cdot 100$,

$\chi=\frac{R_{\mathrm{wp}}}{R_{\exp }}$

with $y_{i}(\mathrm{obs})$ and $y_{i}$ (calc) the observed and calculated intensity at profile point $i$, respectively, and $w_{\mathrm{i}}$ the corresponding, calculated weight.

\subsection{Electronic structure calculations}

The first-principles calculations were performed with the Vienna Ab initio Simulation Package (VASP), ${ }^{54}$ using the projector-augmented wave (PAW) method to describe the electron-ion interactions. ${ }^{55,56}$ Specifically, the $\mathrm{O} 2 s^{2} 2 p^{4}$ and $\mathrm{U} 6 s^{2} 6 p^{6} 5 f^{3} 6 d^{1} 7 s^{2}$ electrons are treated as valence electrons in the PAW potentials used. The energy cut-off for the plane wave basis set was $600 \mathrm{eV}$. As previous authors, we describe the exchange-correlation interactions within the $\mathrm{PBE}+U$ approximation, as applied by Dudarev et al. ${ }^{23}$ Here, only the difference between the parameters describing the screened Coulomb and exchange interactions is relevant, resulting in an effective repulsion parameter $U_{\text {eff }}=U-J$. The values used are $U=4.50 \mathrm{eV}$ and $J=0.54 \mathrm{eV}$, as deduced from photoemission measurements on $\mathrm{UO}_{2} \cdot{ }^{57}$ To overcome the problem of possible metastable solutions, we applied the so-called ramping method, ${ }^{58}$ where the $U_{\text {eff }}$ parameter was adiabatically increased from 0 to the target value of $3.96 \mathrm{eV}$ in 20 steps. Furthermore, we included spin-orbit coupling in all our calculations. The inclusion of spin-orbit configuration interactions partly lifts the degeneracy of the $5 f^{2}$ manifold of $\mathrm{U}(\mathrm{IV})$ and of the $5 f$ manifold of U(V), effectively improving the convergence to the ground state. The effect is important both for describing valence correlation effects and the spin-orbit polarization of the outer-core orbitals.

The crystallographic data used as input for these calculations are those obtained from the neutron diffraction measurements at $1.5 \mathrm{~K}$, reported and discussed in detail in this paper further down. They correspond to the large, tetragonal unit cell $(12.0018 \times 12.0018 \times$ $\left.16.6239 \AA^{3}\right)$ containing 200 atoms $\left(\mathrm{U}_{60} \mathrm{O}_{140}\right)$. For the self-consistency calculations, the Brillouin zone was sampled using a $\Gamma$-centered $4 \times 4 \times 3$ Monkhorst-Pack k-point grid and the total energy was converged to within $10^{-5} \mathrm{eV}$. For the density of states calculations, the tetrahedron method with Blöchl corrections was used and the k-point grid was increased to 
$8 \times 8 \times 6 .{ }^{59}$ The path in $\mathbf{k}$-space for the band structure calculations follows a standard sequence ${ }^{60}$ For charge analysis, we considered the Bader partitioning using the software developed by Henkelman and co-workers, ${ }^{61}$ as well as the Tkatchenko-Scheffler iterative Hirshfeld partitioning, ${ }^{62}$ as implemented in VASP. 


\section{Results}

\subsection{Definitions of the structural model}

The $\mathrm{U}_{3} \mathrm{O}_{7}$ crystal structure described in the $P 4_{2} / n$ space group consists of a unit cell spanned by lattice vectors here denoted as A, B, C. It contains 60 uranium and 140 oxygen atoms, leading to the composition $\mathrm{U}_{60} \mathrm{O}_{140}$ (simplified as $\mathrm{U}_{3} \mathrm{O}_{7}$ ). The expanded cell can also be regarded as 15 subcells, of which 11 have a fluorite-type composition $\left(\mathrm{U}_{4} \mathrm{O}_{8}\right)$, and 4 contain a cuboctahedral oxygen cluster $\left(\mathrm{U}_{4} \mathrm{O}_{13}\right) \cdot{ }^{39}$ In Figure 1, a visualization of the expanded unit cell is presented, illustrating the occurrence of cuboctahedral oxygen clusters on the cell edges and face-centers. Also, a single fluorite-type subcell is visualized by the gray dashed lines. The lattice vectors of the subcell are here denoted as $\mathbf{a}_{\mathbf{f}}, \mathbf{b}_{\mathbf{f}}, \mathbf{c}_{\mathbf{f}}$, and their magnitudes refer to the values which have often been quoted in earlier literature (i.e. $a_{\mathrm{f}}=b_{\mathrm{f}} \approx 5.38 \AA, c_{\mathrm{f}} \approx 5.55 \AA$; $\left.c_{\mathrm{f}} / a_{\mathrm{f}} \approx 1.03\right){ }^{12,63,64}$ The relation between both vector spaces was worked out by Leinders and coworkers: $\mathbf{A}=\mathbf{a}_{\mathbf{f}}-2 \mathbf{b}_{\mathbf{f}}, \mathbf{B}=2 \mathbf{a}_{\mathbf{f}}+\mathbf{b}_{\mathbf{f}}, \mathbf{C}=3 \mathbf{c}_{\mathbf{f} .}{ }^{39}$

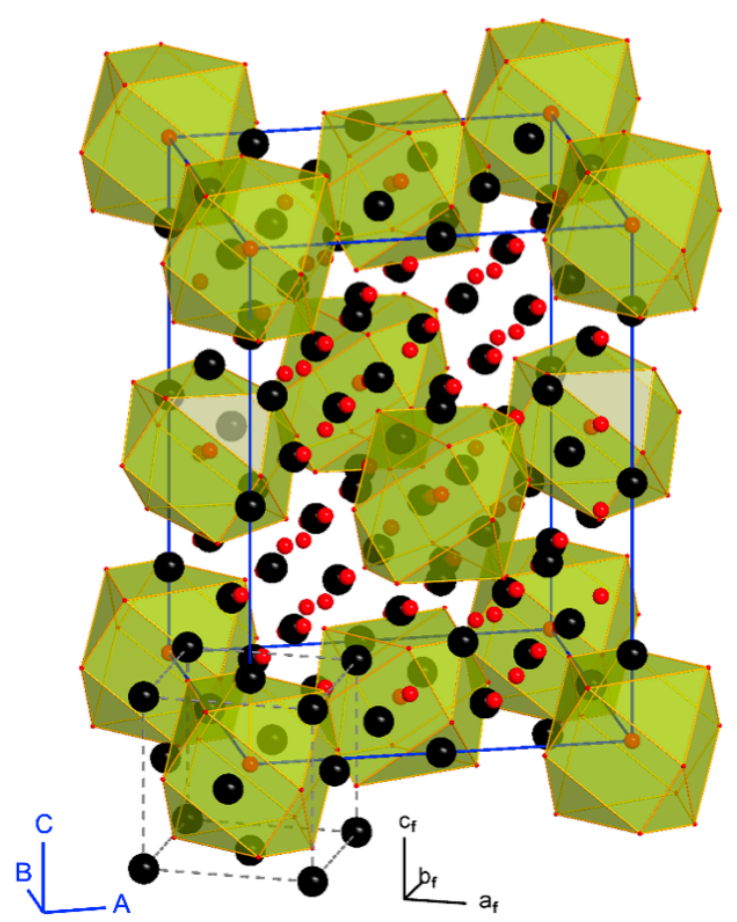

Figure 1. Illustration of the $\mathrm{U}_{3} \mathrm{O}_{7}\left(\mathrm{U}_{60} \mathrm{O}_{140}\right)$ crystal structure trial model derived from electron diffraction data, figure adapted from Leinders et al. ${ }^{39}$ Copyright 2016 American Chemical Society. Uranium and oxygen atoms are represented by black and red spheres, respectively. Green colored polyhedra indicate the positions of cuboctahedral oxygen clusters.

The atomic arrangement of the $\mathrm{U}_{60} \mathrm{O}_{140}$ crystal structure in the $P 4_{2} / n$ space group, as derived from X-ray diffraction and electron diffraction investigations, ${ }^{39}$ is reproduced in Table $\mathrm{S} 1$ of the ESI. Three atomic groups can be distinguished: (1) cations on eight unique Wyckoff sites labeled U1 to U8, (2) anions on 15 fluorite-type positions labeled O1 to O15, (3) anions comprising the cuboctahedral oxygen clusters ( 7 unique Wyckoff sites labeled O'c and O'1 to O'6). The notation originally used to distinguish the size and degree of distortion of the 
cuboctahedra, i.e. parameters $u$ and $\delta$, respectively, was maintained. Table S2 in the ESI shows the atomic coordinates when $u=0.41$ and $\delta=0.0625$. This setting is further referred to as the "trial model".

To evaluate the absence or presence of cuboctahedral oxygen clusters from the diffraction data it is also useful to consider a model which consists simply of 15 fluorite-type subcells $\left(\mathrm{U}_{4} \mathrm{O}_{8}\right)$ in the expanded unit cell. The corresponding structural model is referred to as the parent structure, and its unit cell composition is equal to $\mathrm{U}_{60} \mathrm{O}_{120}$ (simplified as $\mathrm{UO}_{2}$ ). This setting is presented in Table $\mathrm{S} 3$ of the ESI.

\subsection{Sample characterization from diffraction data}

The phase purity of the $\mathrm{U}_{3} \mathrm{O}_{7}$ powder sample was checked by performing X-ray diffraction analysis at room temperature. The XRD pattern presented in Figure 2 (a) obeys the same extinction conditions as for a face-centered cubic (fluorite) structure, but clearly shows tetragonal splitting of all $(h k l)$ reflections, except when $h=k=l$. No satellite reflections can be distinguished, and hence, the pattern can be evaluated directly from the parent structure model with cations and anions located at the corresponding fluorite-type atomic positions (cf. Table S3 in the ESI). The fit to the X-ray pattern based on a Rietveld refinement using the parent structure model as input, is illustrated on Figure 2 (a). It shows relatively good agreement $\left(R_{\mathrm{wp}}=10.40, \chi=3.04\right)$, although some discrepancies in the fitted intensity (e.g. around $10 \%$ overfitting of the $(111)_{\text {f }}$ reflection) are apparent. Lattice parameters and agreement factors obtained from the refinement are reported in Table 1.

In addition to room temperature X-ray diffraction, the sample was also analyzed by room temperature neutron diffraction (ND). A relevant section of the acquired ND pattern is presented in Figure 2 (b). To allow direct comparison with the aforementioned XRD pattern, the abscissa of both figures was set to the $d$-spacing (in unit $\AA$ ), but additionally, both subplots show the corresponding Bragg angles on a separate horizontal axis.

The ND pattern contains high-intensity reflections which originate from the fluorite-type parent structure of $\mathrm{U}_{3} \mathrm{O}_{7}$. Note that, unlike for X-rays, neutron scattering factors for uranium and oxygen are similar, hence reflections of the type $(h k l)_{\mathrm{f}}$ with $h+k+l=4 n+2$ are very weak in the neutron diffractograms due to out-of-phase diffraction of uranium and oxygen atoms. In addition to the sharp, parent structure reflections, a series of satellite reflections can be readily distinguished in the region between $35-50^{\circ} 2 \theta$. Also, the background appears more perturbed, which is caused by the occurrence of numerous, low-intensity satellite reflections. Similarly to the evaluation of the XRD pattern, the ND pattern was evaluated via Rietveld refinement, using the parent structure model (results are presented in Table 1). The resulting fit (solid red line in Figure $2(\mathrm{~b}), R_{\mathrm{wp}}=13.39, \chi=4.82$ ) also contains discrepancies in the fitted intensity, and obviously, it captures only reflections corresponding to the fluorite-type parent structure. This allows to distinguish the satellite reflections from the main reflections. 


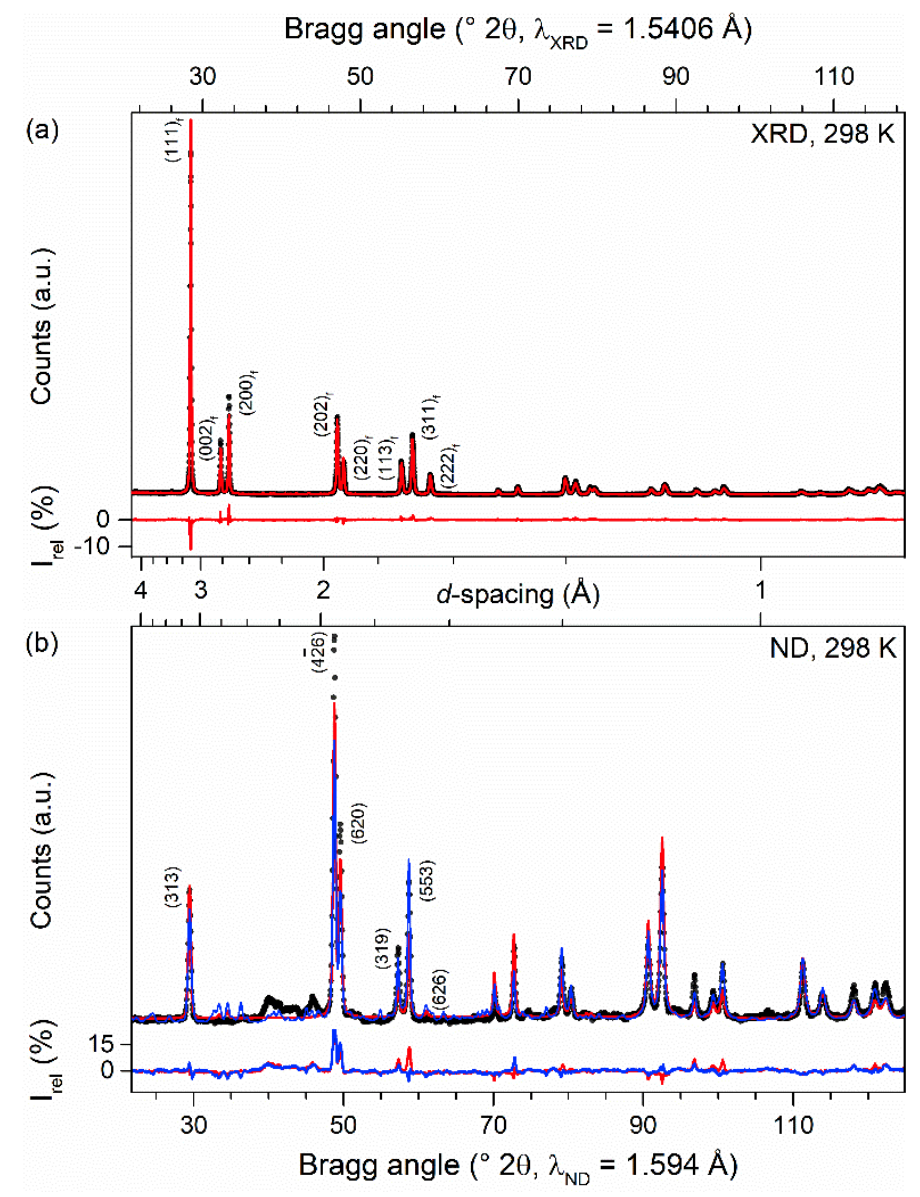

Figure 2. Room temperature X-ray (a) and neutron (b) diffractograms of a polycrystalline $\mathrm{U}_{3} \mathrm{O}_{7}$ powder sample. Measured data are represented by black dots. Solid lines represent Rietveld fits to the pattern using either the parent structure without oxygen clusters (red line), or the trial model with cuboctahedral oxygen clusters (blue line). The corresponding difference profiles are shown below each subplot on a relative intensity $\left(\mathrm{I}_{\mathrm{rel}}\right)$ scale. Miller indices of some of the reflections are given either with respect to basis vectors $\mathbf{a}_{\mathbf{f}}, \mathbf{b}_{\mathbf{f}}, \mathbf{c}_{\mathbf{f}}$ of the original fluorite structure (subplot a) or basis vectors A, B, C of the expanded cell (subplot b).

Table 1. Agreement factors and lattice parameter values obtained after Rietveld refinement of the room temperature XRD and neutron diffraction patterns. Estimated standard deviations on the trailing digit (esd's) are given in parentheses.

\begin{tabular}{llll}
\hline $\begin{array}{l}\text { Diffractogram } \\
\text { Structural model }\end{array}$ & $\begin{array}{l}\text { XRD, 298 K } \\
\text { Parent }\end{array}$ & $\begin{array}{l}\text { ND, 298 K } \\
\text { Parent }\end{array}$ & $\begin{array}{l}\text { ND, 298 K } \\
\text { Trial }\end{array}$ \\
\hline $\boldsymbol{R}_{\mathrm{wp}}(\%)$ & 10.40 & 13.39 & 13.39 \\
$\chi$ & 3.04 & 4.82 & 4.82 \\
$\boldsymbol{A}=\boldsymbol{B}(\AA)$ & $12.0303(2)$ & $12.0258(8)$ & $12.0258(8)$ \\
$\boldsymbol{C}(\AA)$ & $16.6542(3)$ & $16.651(1)$ & $16.650(2)$ \\
$\boldsymbol{a}_{\mathbf{f}}=\boldsymbol{b}_{\mathrm{f}}(\AA)$ & $5.3801(1)$ & $5.3781(4)$ & $5.3781(4)$ \\
$\boldsymbol{c}_{\mathrm{f}}(\AA)$ & $5.5514(1)$ & $5.5503(3)$ & $5.5500(6)$ \\
$\boldsymbol{c}_{\mathrm{f}} / \boldsymbol{a}_{\mathrm{f}}$ & 1.032 & 1.032 & 1.032 \\
\hline
\end{tabular}


It is evident that the parent structure model does not allow a correct evaluation of the ND data. The satellite reflections in the diffraction pattern relate to the occurrence of oxygen clusters forming a long-range order in the crystal structure of $\mathrm{U}_{3} \mathrm{O}_{7}{ }^{13,39}$ Hence, a structural model which includes oxygen clusters needs to be applied. Subsequently, Rietveld refinement was performed using the previously defined trial model (see section §3.1). However, the resulting fit (solid blue line in Figure $2(\mathrm{~b}), R_{\mathrm{wp}}=13.39, \chi=4.82$ ) does not improve, see also Table 1. Although additional reflections are modelled in the $35-50^{\circ} 2 \theta$ range, significant misfits in the profiles of both the satellite and main reflections occur. Clearly, a further refinement of atomic arrangements and displacement parameters in the expanded structural model is required. For this purpose, we will consider neutron diffraction data sets acquired both at room temperature and at $1.5 \mathrm{~K}$ (see sections $\S 3.3$ and 3.4).

\subsection{Assessment of low-temperature antiferromagnetic order in $\mathrm{U}_{3} \mathrm{O}_{7}$}

The acquisition of diffraction data at cryogenic temperatures results in minimal interference of thermal displacements on local disorder, in contrast to conventional room temperature acquisition. However, cryogenic conditions may also affect the crystal structure by inducing a phase transition or allowing additional magnetic ordering schemes. Such behavior, and in particular an antiferromagnetic transition, has been reported to occur in $\mathrm{UO}_{2}$ at temperatures just above $30 \mathrm{~K} .{ }^{65,66}$ To identify or rule out such effects on $\mathrm{U}_{3} \mathrm{O}_{7}$, neutron diffraction experiments were first carried out using the high-intensity two-axis diffractometer at beamline D20 of the ILL, operating at $1.5 \mathrm{~K}$ and $100 \mathrm{~K}$ in a He cryostat (see details in $§ 2.2$ ).

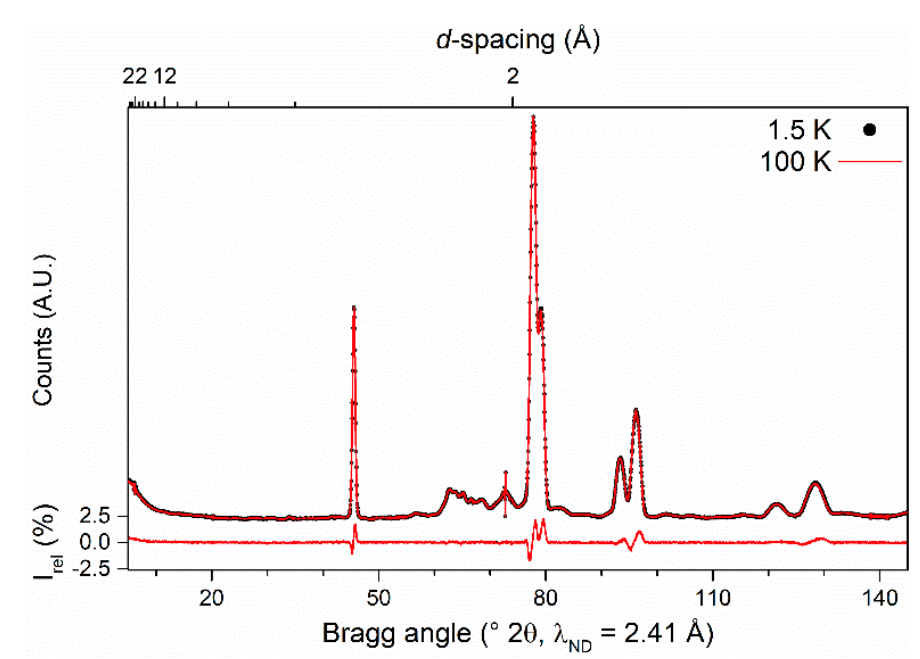

Figure 3. Neutron diffractograms of polycrystalline $\mathrm{U}_{3} \mathrm{O}_{7}$ powder acquired at $1.5 \mathrm{~K}$ (black dots) and $100 \mathrm{~K}$ (red line) using the high-intensity two-axis diffractometer at beamline D20 $\left(\lambda_{\mathrm{ND}}=2.41 \AA\right)$ of the ILL. Intensities are normalized to the main reflection. The difference profile between both patterns is shown at the bottom of the plot on a relative intensity ( $\left.\mathrm{I}_{\mathrm{rel}}\right)$ scale. No indications for long-range antiferromagnetic ordering could be distinguished from the data comparison.

A comparison between both scans, with intensity normalized to the highest intensity reflection, is presented in Figure 3. The difference profile is additionally shown at the bottom 
of the plot, on a relative intensity scale. Some scatter is visible around the main reflections due to differences in thermal expansion, but no additional reflections can be distinguished in the low angular range. Based on these data, a phase transition producing a long-range antiferromagnetic order between $1.5 \mathrm{~K}$ and $100 \mathrm{~K}$ can be ruled out. On the other hand, a canted magnetic order which does not produce strong additional scattering signals cannot be immediately ruled out, and such an assessment will be carried out based on the electronic structure calculations reported and discussed further.

\subsection{Structure refinement at $1.5 \mathrm{~K}$ and $298 \mathrm{~K}$}

To comprehensively assess the $\mathrm{U}_{3} \mathrm{O}_{7}$ crystal structure, neutron diffraction data were additionally measured using the high-resolution two-axis diffractometer of beamline D2B at the ILL, both at $1.5 \mathrm{~K}$ and $298 \mathrm{~K}$ (see details in $\$ 2.2$ ). Apart from the thermal contraction and reduced diffuse scatter, the $1.5 \mathrm{~K}$ neutron diffraction pattern (displayed in Figure 4 (a)) is identical to the diffractogram recorded at room temperature, i.e. no additional reflections are observed.
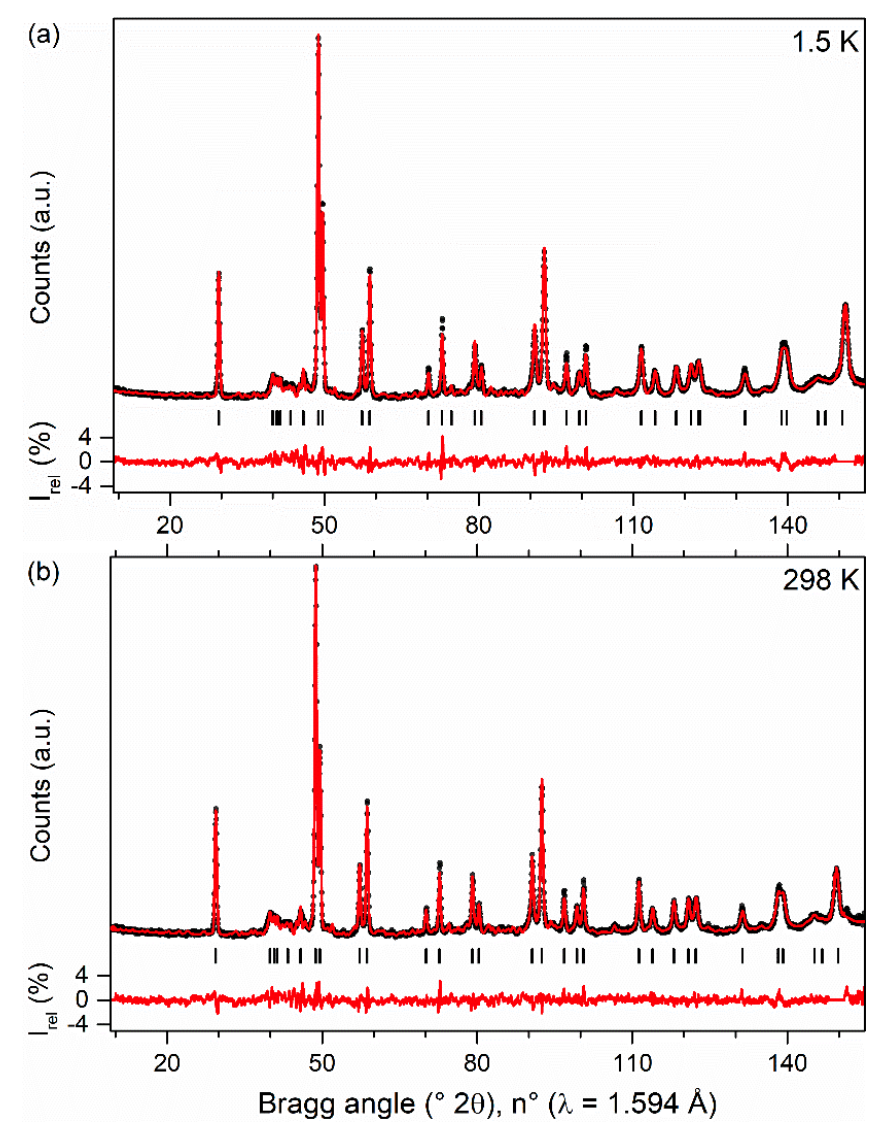

Figure 4. $\mathrm{U}_{3} \mathrm{O}_{7}$ neutron diffractograms, acquired at $1.5 \mathrm{~K}$ (top) and $298 \mathrm{~K}$ (bottom). Measured data points are represented by black dots, a fit to the pattern after Rietveld refinement is shown by the overlaying red line. The corresponding difference profile is shown below the pattern, on a relative intensity $\left(\mathrm{I}_{\text {rel }}\right)$ scale. Vertical black lines denote the position of reflections with a relative intensity larger than $2 \%$. 
The $1.5 \mathrm{~K}$ neutron diffractogram was analyzed by Rietveld refinement, following the general approach outlined in section $\S 2.2$. The scan range considered was from 9.00 to $159.95^{\circ} 2 \theta$, but a window between 149.5 and $153.0^{\circ} 2 \theta$ was excluded because of a parasitic signal from the cryostat. The parameter set was progressively expanded by refining four individual isotropic thermal displacement parameters $\left(u_{\mathrm{U} 1-8}, u_{\mathrm{O} 1-15}, u_{\mathrm{O}}{ }^{\prime} \mathrm{c}, u_{\mathrm{O}}{ }^{\prime} 1-6\right)$, each constraining a subgroup of atoms in the structural model, corresponding respectively to U1-U8, O1-O15, O'c, and O'1-O'6. This refinement improved already $\left(R_{\mathrm{wp}}=9.07\right.$ and $\left.\chi=4.17\right)$ as compared to the parent structure refinement reported in section $\S 3.1$. However, a significant misfit on the intensities of the satellite reflections was still apparent, and additionally, the isotropic thermal displacement parameters were still elevated $\left(u_{\mathrm{U} 1-8}=0.0090(5), u_{\mathrm{O} 1-15}=0.0319(7)\right.$, $\left.u_{\mathrm{O}}{ }^{\prime} \mathrm{c}=0.15(4), u_{\mathrm{O}}{ }_{1-6}=0.141(9)\right)$. At the temperature under investigation this is indicative of further structural disorder, mainly on the oxygen sublattice.

In a next refinement cycle the coordinates of atoms on general (xyz) Wyckoff positions, and subsequently, those of the remaining atoms were included, constrained to their respective site symmetry conditions. This operation led to a remarkably better agreement between the diffraction data and fit $\left(R_{\mathrm{wp}}=4.82\right.$ and $\chi=2.25$, see Figure $\left.4(\mathrm{a})\right)$, and also the isotropic thermal displacement parameters decreased significantly $\left(u_{\mathrm{U} 1-8}=0.0040(6)\right.$, $\left.u_{\mathrm{O} 1-15}=0.0085(9), u_{\mathrm{O}^{\prime} \mathrm{c}}=0.05(1), u_{\mathrm{O}^{\prime} 1-6}=0.029(2)\right)$. A summary of the refinement results is presented in Table 2.

Table 2. Comparison of results obtained after Rietveld refinement of the $1.5 \mathrm{~K}$ and $298 \mathrm{~K}$ neutron diffraction data. Esd's are given in parentheses.

\begin{tabular}{llcc}
\hline & & \multicolumn{2}{c}{ Acquisition temperature } \\
\cline { 3 - 4 } & & $\mathbf{1 . 5} \mathbf{~ K}$ & $\mathbf{2 9 8 ~ K}$ \\
\hline $\boldsymbol{R}_{\mathbf{w p}}(\%)$ & & 4.82 & 4.95 \\
$\chi$ & & 2.25 & 1.80 \\
$\boldsymbol{A}=\boldsymbol{B}(\AA)$ & & $12.0018(3)$ & $12.0253(3)$ \\
$\boldsymbol{C}(\AA)$ & & $16.6239(7)$ & $16.6496(6)$ \\
& U1-8 & $0.0040(6)$ & $0.0070(6)$ \\
& O1-15 & $0.0085(9)$ & $0.014(1)$ \\
$\boldsymbol{u}_{\text {iso }}\left(\AA^{2}\right)$ & O'c & $0.05(1)$ & $0.05(1)$ \\
& O'1-6 & $0.029(2)$ & $0.034(3)$ \\
\hline
\end{tabular}

The $298 \mathrm{~K}$ neutron diffraction data acquired at beamline D2B was subsequently evaluated via Rietveld refinement, using the crystallographic information obtained at $1.5 \mathrm{~K}$ as starting input data. The scan range considered was from 9.00 to $154.95^{\circ} 2 \theta$, with a window between 148 and $151^{\circ} 2 \theta$ being excluded because of the previously discussed parasitic scattering signal coming from the cryostat. The refinement approach was identical as before, also including the 
four individual isotropic thermal displacement parameters $\left(u_{\mathrm{U} 1-8}, u_{\mathrm{O} 1-15}, u_{\mathrm{O}}{ }^{\prime} \mathrm{c}, u_{\mathrm{O}}{ }^{\prime} 1-6\right)$ defined earlier. The calculation converged readily, demonstrating the suitability of the input structure, and excellent agreement was obtained $\left(R_{\mathrm{wp}}=4.95\right.$ and $\left.\chi=1.80\right)$, see Figure $4(\mathrm{~b})$. Because the diffraction data was acquired at room temperature, the isotropic thermal displacement parameters increased slightly, as compared to their values at $1.5 \mathrm{~K}$ (see Table 2).

The thermal displacement parameters of the oxygen atoms comprising the cuboctahedral oxygen clusters (O'c and O'1-6) stand out from the values of the U1-8 and O1-15 atoms at both acquisition temperatures. With respect to the $1.5 \mathrm{~K}$ conditions, this is indicative of some remaining structural disorder. Arguably, it could be possible to release the constraint on $u_{\mathrm{O}}{ }^{\prime} 1-6$ and continue the refinement using anisotropic parameters on $\mathrm{O}^{\prime} \mathrm{c}$ and the six individual $\mathrm{O}$ ' atoms. However, such operation would increase the total number of variables from 85 to 125 . We attempted this model, but obtained no smooth convergence in the refinement procedure, most likely because of the strong correlation between positional and anisotropic parameters of the atomic model. In another attempt, the positional parameters were kept fixed, and only the thermal displacement parameters were further refined, but this led to meaningless negative values for the anisotropic ellipsoids axes, which indicates we overreached the information of the diffraction data set.

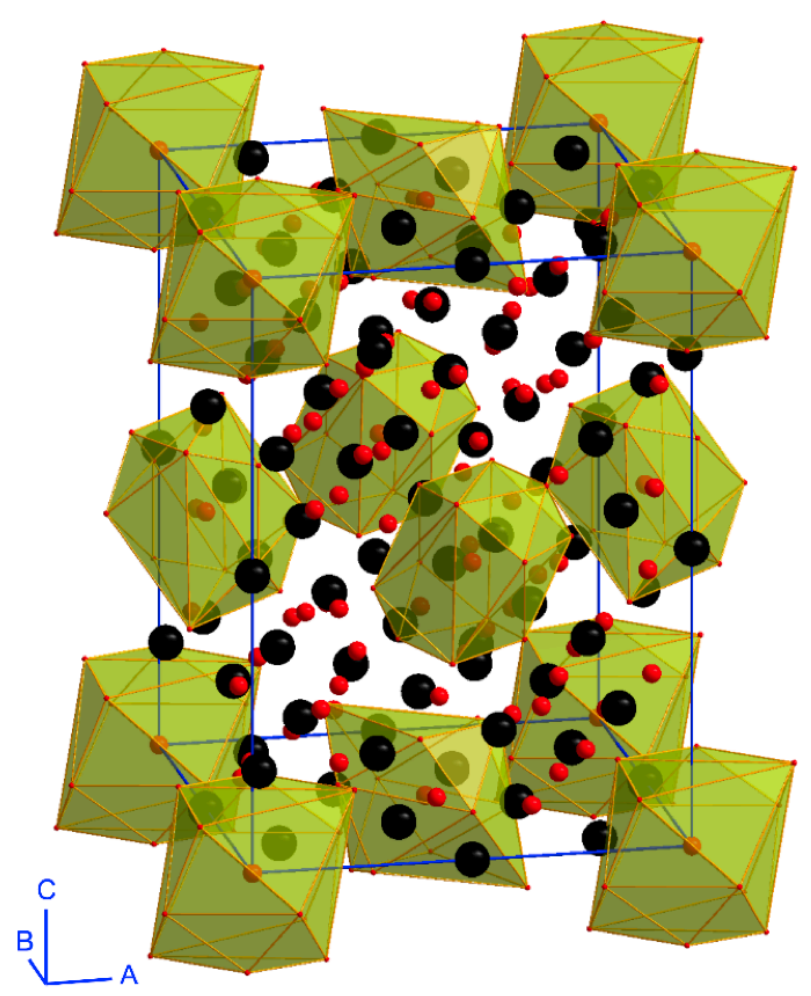

Figure 5. Illustration of the refined $\mathrm{U}_{3} \mathrm{O}_{7}$ crystal structure at $1.5 \mathrm{~K}$, in the expanded unit cell $\left(P 4_{2} / n\right)$ with lattice parameters $A=B=12.0018(3) \AA, C=16.6239(7)$. Uranium and oxygen atoms are represented by black and red spheres, respectively. Green colored polyhedra indicate the positions of distorted cuboctahedral oxygen clusters. 
All structural parameters refined from the $1.5 \mathrm{~K}$ and $298 \mathrm{~K}$ diffraction data are summarized in their corresponding crystallographic information files (CIF), and made available as supporting information. Additionally, comprehensive overviews of the refined structural parameters are presented in Tables S4 and S5 of the ESI. A visualization of the expanded unit cell, refined from the $1.5 \mathrm{~K}$ neutron diffraction data, is shown in Figure 5. It appears that the uranium atoms remain close to their original fluorite-type positions, with relative displacements not exceeding $0.16 \AA$. The oxygen atoms, however, are much more severely affected, and variations from the original positions may reach up to $0.89 \AA$.

One of the most obvious differences with the initial trial model (see Figure 1) is that the cuboctahedral oxygen clusters are no longer regular. For a regular cuboctahedron, the vertices are described by a $<u u 0>$-type displacement from the central atom (12 symmetry-equivalent positions). ${ }^{67,68}$ Hence, the value of $u$ denotes the cuboctahedron size, and in previous studies where regular cuboctahedral oxygen clusters were assumed to occur in $\mathrm{U}_{3} \mathrm{O}_{7}$, values between 0.37 and 0.41 have been derived. ${ }^{38,42,69}$ Although we now find that the clusters are severely distorted, a corresponding value of the $u$ parameter may be derived by averaging the distance between the polyhedron vertices and the center. Based on the model refined on the $1.5 \mathrm{~K}$ neutron diffraction data, an average value of $u=0.39 \pm 0.04(1 \sigma)$ is obtained, which is in excellent agreement with earlier predictions.

\subsection{Electronic structure calculations}

The electronic properties of $\mathrm{U}_{3} \mathrm{O}_{7}$, imposed by the refined structural model at $1.5 \mathrm{~K}$, were assessed with emphasis on the environment of the uranium atoms and their related properties. Given that long-range collinear magnetic order has been ruled out from our neutron scattering experiments (see section §3.3), the possible emergence of canted magnetic order induced by spin-orbit coupling (SOC) was considered. Figure 6 shows the band structure of $\mathrm{U}_{3} \mathrm{O}_{7}$ obtained including SOC, with energies referenced to the valence band maximum. It indicates that $\mathrm{U}_{3} \mathrm{O}_{7}$ is a semiconductor, with a band gap close to $0.32 \mathrm{eV}$. Alternatively, a paramagnetic calculation neglecting SOC predicted $\mathrm{U}_{3} \mathrm{O}_{7}$ to be a metal (see Figure $\mathrm{S} 1$ in the ESI), contrary to experimental evidence. ${ }^{70}$ Another discrepancy, which occurs when SOC is excluded from calculations, is related to the occupation of the $5 f$ orbitals. The nuclear model contains eight groups of uranium atoms (U1, U2, .. U8), and although no point symmetry was enforced in the calculations, the SOC correction appears only weakly effective in breaking the tetragonal symmetry of the nuclear model. Hence, eight groups of uranium atoms showing similar properties are reproduced (see Figure S2 in the ESI). On the other hand, results of calculations without SOC are not satisfactory because they show occupancy discrepancies within most of the groups. Therefore, the latter type of calculation is unable to describe properly the $5 f$ nature of the states in $\mathrm{U}_{3} \mathrm{O}_{7}$, and henceforth, only results including SOC are reported and discussed. We point out that the relevance of SOC to the chemistry of uranium complexes (and more generally, actinide complexes) has been long recognized, ${ }^{71}$ and furthermore, it is of 
paramount importance to explore non-collinear magnetic configurations, as will be demonstrated in section $\$ 4.2$.

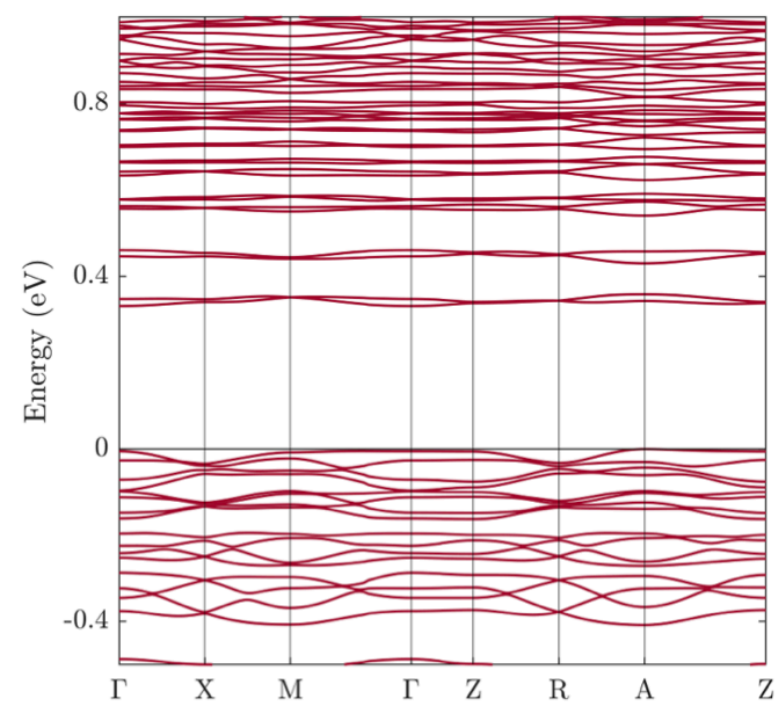

Figure 6. $\mathrm{PBE}+U$ calculated band structure of $\mathrm{U}_{3} \mathrm{O}_{7}$ along the high symmetry lines of the Brillouin zone that corresponds to the tetragonal structure, including spin-orbit coupling. $\mathrm{U}_{3} \mathrm{O}_{7}$ is predicted to be a semiconductor with a band gap of close to $0.32 \mathrm{eV}$ (energies referenced to the valence band maximum).

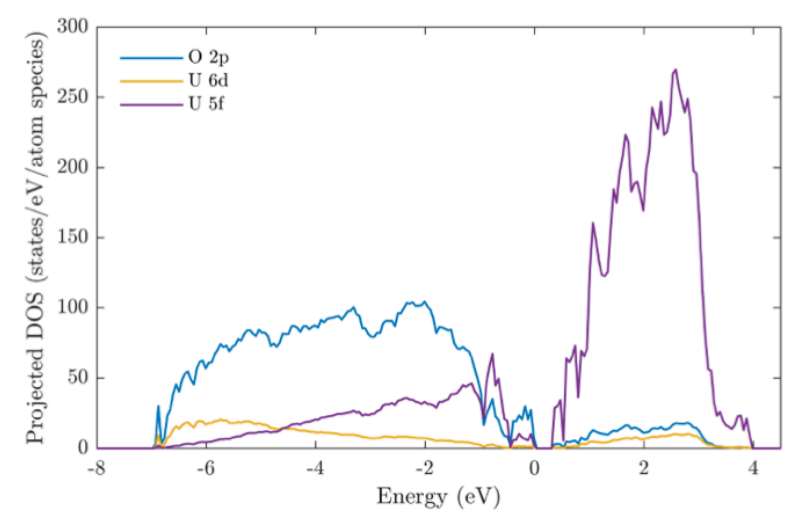

Figure 7. $\mathrm{PBE}+U$ projected density of states of $\mathrm{U}_{3} \mathrm{O}_{7}$. The dominant contributions in the energy range shown come from the oxygen $2 p$ and the uranium $6 d$ and $5 f$ states. Interestingly, the oxygen $2 p$ states contribute more to the top of the valence band than the uranium $5 f$ states. This implies that $\mathrm{U}_{3} \mathrm{O}_{7}$ can be considered a charge-transfer insulator, and not strictly a Mott insulator like $\mathrm{UO}_{2}$ (energies referenced to the valence band maximum).

The electronic structure of $\mathrm{U}_{3} \mathrm{O}_{7}$ was further analyzed by considering the nature of the electronic states in the valence and conduction bands. Figure 7 shows the projected character of the corresponding contributions. The valence band has main contributions from oxygen $2 p$ and uranium $5 f$ states, and a lesser presence of uranium $6 d$ states. The conduction band is dominated by uranium $5 f$ states, with minor contributions of oxygen $2 p$ and uranium $6 d$ states. This is rather similar to what is observed in $\mathrm{UO}_{2} .{ }^{27}$ Interestingly, whereas in $\mathrm{UO}_{2}$ the gap occurs essentially between uranium $5 f$ states, i.e. the top of the valence band and bottom of 
the conduction bands are mainly of uranium $5 f$ character, in $\mathrm{U}_{3} \mathrm{O}_{7}$ the valence band is mainly of oxygen $2 p$ character. This means that in contrast to $\mathrm{UO}_{2}$, which is a Mott insulator, $\mathrm{U}_{3} \mathrm{O}_{7}$ is better described as a charge-transfer insulator, ${ }^{16}$ or rather as a mix of the two, with a stronger bias toward the latter. The difference with $\mathrm{UO}_{2}$ can be understood as arising from the overall change in oxidation state of the uranium atoms, which in turn also produce changes of the coordination polyhedra and shorter $\mathrm{U}-\mathrm{O}$ bonds.

The uranium chemical states in the refined structural model of $\mathrm{U}_{3} \mathrm{O}_{7}$ were subsequently evaluated in two different ways. Firstly, via the Bader (Atoms in Molecules) analysis, where the atomic charge is integrated within non-overlapping atomic basins having boundaries defined by the zero-flux condition of the electron density. ${ }^{72}$ And secondly, via the iterative Hirshfeld (Hirshfeld-I) approach, in which the charge density is mapped over a reference density and it is split in basins that can partially overlap (the iterative Hirshfeld method is an improved version of the original Hirshfeld partitioning). ${ }^{62}$ In principle, the Hirshfeld-I charges correlate with the ion interactions and provide a better descriptor of the nominal oxidation state of an ion. ${ }^{62}$

The results of Bader and Hirshfeld-I charge analyses are presented in Figure 8. The eight uranium groups are clearly reproduced, and within each group the values are consistent with the site symmetry rules. The independent calculation of the Bader and Hirshfeld-I charges for each atom in the cell provides proof of the accuracy of the calculations, which are dependent on the sampling over a real space fine grid and on the energy cutoff of the basis functions. We can distinguish at least three families of environments: (1) Uranium site U1 shows the highest charge depletion, and hence, must exhibit the highest oxidation state, (2) sites U3, U4, U5, U7 fall within a narrow charge interval and will have a similar but lower oxidation state than U1, (3) sites U2, U6, U8 are assigned the lowest charges, but display some scatter.

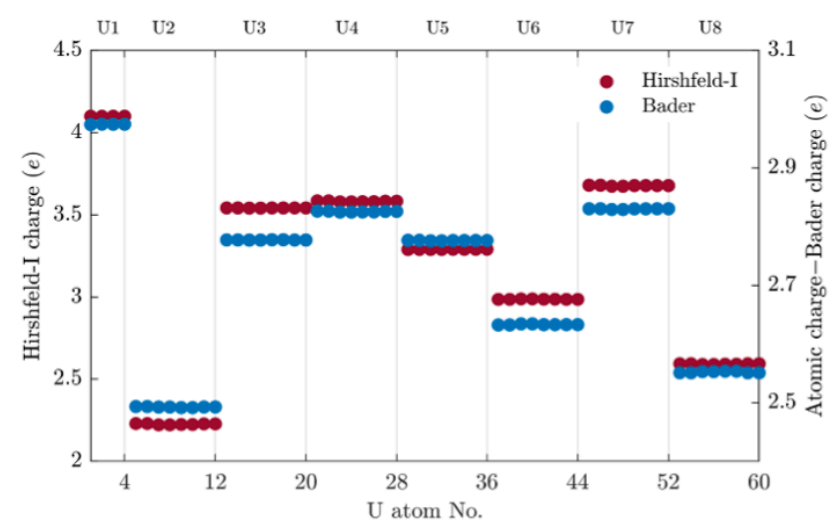

Figure 8. Uranium atom charges resulting from the Iterative-Hirshfeld (Hirshfeld-I) and Bader partitioning schemes. Both methods show a similar trend from which at least three types of environments can be deduced: U1, (U3, U4, U5, U7), and (U2, U5, U8). 
The non-collinear spin-resolved calculations of the electronic structure allow also the complete determination of the amplitude and the orientation of the local magnetic moment of the different uranium atoms. Due to the localized $5 f$ orbitals one can expect a localized magnetic moment, although its magnitude and direction will depend on the actual electronic structure. In Figure 9 the magnitude of the spin magnetic moment is presented, taking into account the contribution of either all orbitals (dark red dots) or only the $5 f$ orbitals (gold dots). This comparison shows clearly that the dominant contribution stems from the $5 f$ orbitals. Interestingly, the magnetic moment values reproduce very distinctly the aforementioned three families of uranium sites. Quite importantly, uranium atoms at Wyckoff site $4 d$ (i.e. atoms U1) are found to have a (nearly) zero magnetic moment, which would correspond to a formal hexavalent state (absence of $5 \mathrm{f}$ electrons). The highest magnetic moments are assigned to uranium sites $\mathrm{U} 2$, U6 and U8 (1.6 $\mu_{\mathrm{B}}$ on average), whereas an intermediate value $\left(0.8 \mu_{\mathrm{B}}\right.$ on average) is calculated for uranium sites U3, U4, U5 and U7. By combining these results with the charge analyses it may be presumed that the $\mathrm{U}_{3} \mathrm{O}_{7}$ phase contains a single hexavalent environment (U1), four pentavalent environments (U3, U4, U5, U7), and three tetravalent environments (U2, U6, U8). Based on the site symmetry values, this would lead to a $24 / 60 \times \mathrm{U}(\mathrm{IV})+32 / 60 \mathrm{U}(\mathrm{V})+4 / 60 \mathrm{U}(\mathrm{VI})$ distribution of uranium chemical states.

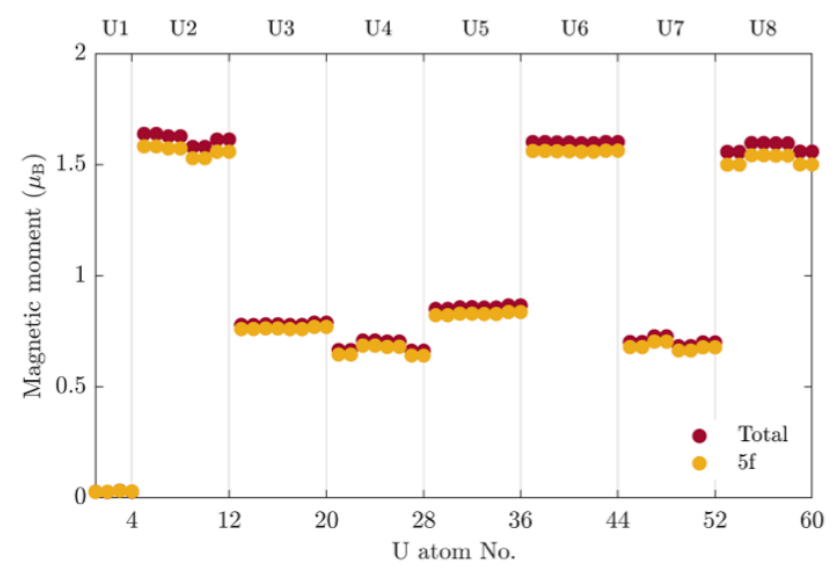

Figure 9. Local magnetic moment of the uranium atoms: total (dark red dots) and $f$-orbitals contribution only (gold dots). The analysis of the magnetic moments clearly partitions the U1U8 atoms into three classes establishing a bijective correspondence with the U(IV), U(V), and U(VI) environments. 


\section{Discussion}

\subsection{Charge localization in the nuclear structure}

Recent experimental evidence, specifically X-ray absorption spectroscopy results, ${ }^{38,47}$ emphasized that tetravalent and pentavalent uranium states are the prevalent charge compensation mechanisms in both $\mathrm{U}_{4} \mathrm{O}_{9}$ and $\mathrm{U}_{3} \mathrm{O}_{7}$. The occurrence of a hexavalent chemical state could only be evidenced in $\mathrm{U}_{3} \mathrm{O}_{8}$ and $\mathrm{UO}_{3}$. Similarly, in a recent theoretical study by Ao and coworkers, Bader charge values corresponding only to tetravalent and pentavalent uranium states were assigned to the $\mathrm{U}_{3} \mathrm{O}_{7}$ phase. ${ }^{3}$ Their calculation, however, pertains to the split quad-interstitial oxygen defect model, and results in different atomic environments and charge distributions which cannot be normalized and compared to the work presented here. Our results coincide in that $\mathrm{U}(\mathrm{IV})$ and $\mathrm{U}(\mathrm{V})$ charges are dominant in the $\mathrm{U}_{3} \mathrm{O}_{7}$ phase, but additionally, a small contribution of U(VI) states is found. To further assess the uranium speciation, bond-valence-sum (BVS) calculations were performed using the well-known Zachariasen parameter set derived from tetra-, penta- and hexavalent uranium compounds. ${ }^{73}$ The results are presented in Table 3.

Table 3. Cation charges derived from bond-valence-sum calculations, applying the Zachariasen parameter set. ${ }^{73}$ For each atomic site, only the calculated value with the smallest deviation from the nominal valence may be retained. Incompatible values are parenthesized and greyed out, for clarity towards the reader. The esd on the BVS values, propagated from the uncertainty on the refined structural data amounts to $\pm 0.4(1 \sigma)$ on average.

\begin{tabular}{|c|c|c|c|c|c|}
\hline & \multirow{2}{*}{$\begin{array}{l}\text { Site } \\
\text { mult. }\end{array}$} & \multicolumn{3}{|c|}{ Bond-valence-sum } & \multirow{2}{*}{$\begin{array}{l}\text { Formal cation } \\
\text { charge assigned }\end{array}$} \\
\hline & & $\mathbf{U}(\mathbf{I V})$ & $\mathbf{U}(\mathbf{V})$ & U(VI) & \\
\hline $\mathbf{U} 1$ & 4 & (7.3) & $(6.8)$ & 6.6 & $4 \times 6^{+}$ \\
\hline $\mathbf{U} 2$ & 8 & 4.1 & $(3.8)$ & $(3.6)$ & $8 \times 4^{+}$ \\
\hline $\mathbf{U} 3$ & 8 & $(5.7)$ & 5.3 & $(5.0)$ & $8 \times 5^{+}$ \\
\hline $\mathbf{U} 4$ & 8 & (4.8) & 4.5 & $(4.3)$ & $8 \times 5^{+}$ \\
\hline U5 & 8 & $(4.8)$ & 4.4 & $(4.2)$ & $8 \times 5^{+}$ \\
\hline U6 & 8 & 4.5 & $(4.2)$ & $(4.0)$ & $8 \times 4^{+}$ \\
\hline $\mathbf{U} 7$ & 8 & $(5.3)$ & 4.9 & $(4.7)$ & $8 \times 5^{+}$ \\
\hline \multirow[t]{3}{*}{ U8 } & 8 & 4.1 & (3.8) & $(3.4)$ & $8 \times 4^{+}$ \\
\hline & & \multicolumn{3}{|c|}{ Average cation charge } & Total cation charge \\
\hline & & \multicolumn{3}{|c|}{4.67} & $280^{+}$ \\
\hline
\end{tabular}

The BVS results reproduce the observations from the electronic state calculations, and divide the associated formal cation charge in three groups of uranium sites: (1) Site U1 displays the highest cation charge and can only be assigned a U(VI) state; (2) sites U3, U4, U5, and U7 correspond best to a $\mathrm{U}(\mathrm{V})$ state, but the calculated charges display some scatter from the 
nominal $5^{+}$value; (3) sites U2, U6, U8 are associated to a U(IV) state. This thesis is further supported by evaluating also the average $(280 / 60=4.67)$ and total cation charge. By taking into account the site multiplicity factors the latter value corresponds exactly to the theoretically expected value to respect Pauling's electrostatic valence rule, i.e. in $\mathrm{U}_{60} \mathrm{O}_{140}$ : $\left(24 \times 4^{+}+32 \times 5^{+}+4 \times 6^{+}\right)=280^{+}=\left(140 \times 2^{-}\right)$.

(a)

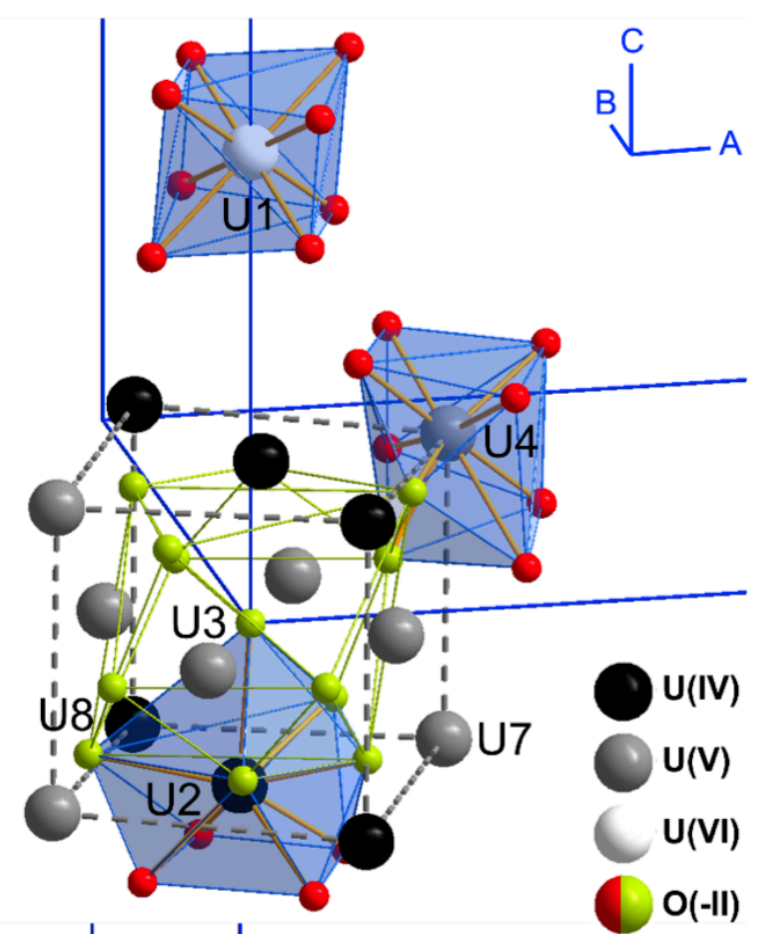

(b)

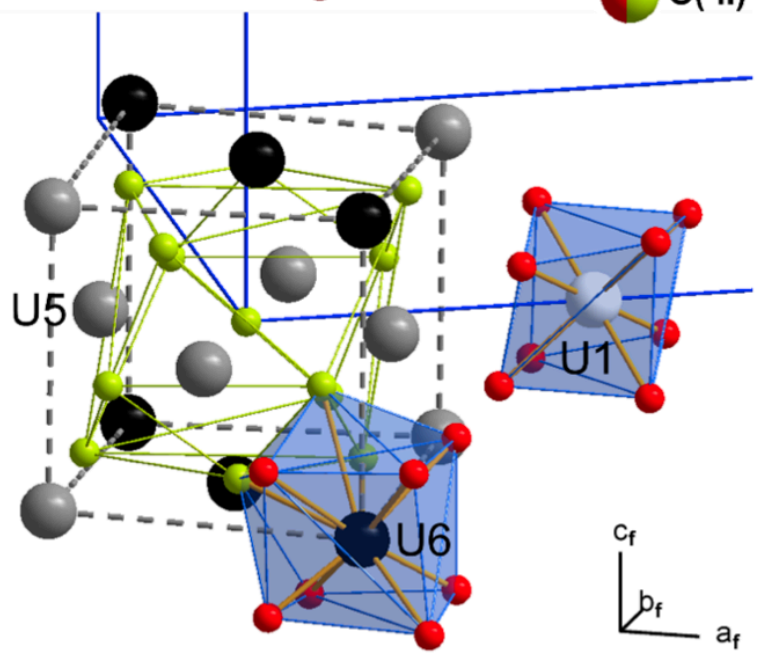

Figure 10. Illustration of some of the uranium coordination environments in the refined $\mathrm{U}_{3} \mathrm{O}_{7}$ structure. Uranium atoms are differentiated by black, dark grey and white colors, corresponding respectively to tetra-, penta-, and hexavalent states. Oxygen atoms are represented by red and green spheres to distinguish between anions on the originally, fluoritetype positions, and those associated to the cuboctahedral clusters, respectively. (a) A 9-fold coordination (capped square-antiprismatic and elongated square pyramidal) occurs around sites U2, U3, U4, U7 and U8. (b) Sites U5 and U6 display a 10-fold coordination (sphenocorona environments). Site U1 is distant from any of the oxygen clusters and exhibits an 8-fold coordination (distorted cubic) with two short "oxo-type" U-O bonds. 
It is now useful to correlate the local coordination chemistry in the $\mathrm{U}_{3} \mathrm{O}_{7}$ structure to the derived uranium speciation, see Figure 10. Nine-fold coordinated uranium atoms (sites U2, $\mathrm{U} 3, \mathrm{U} 4, \mathrm{U} 7$, and U8) are located around the oxygen clusters, and this group can further be subdivided based on their geometry. Sites U2 and U3 are adjacent to the (originally) squarefaces of a neighboring cuboctahedral oxygen cluster and display a capped squareantiprismatic environment. Sites U4, U7 and U8 represent uranium atoms closest to (originally) triangular faces of the cuboctahedra, showing elongated square pyramidal environments. Ten-fold coordination occurs around uranium sites U5 and U6, conforming best to sphenocorona environments. One uranium site (U1) is eight-fold coordinated, but strongly distorted (see also Figure 10) from its original fluorite-type coordination in the trial model. Four pairs of opposite bonds occur (U1-O9, U1-O10, U1-O12, and U1-O15), with the two U1-O9 bonds being very short "oxo-type" bonds (1.93 $\AA$ ). The U1 environment also displays shorter-than-average interatomic distances between oxygen atoms (e.g. $2.21 \AA$ between O9-O15 and $2.38 \AA$ between O9-O15), leading to an overall very compact environment.

Oxo-type bonding has been commonly associated to pentagonal bipyramidal (seven-fold), or octahedral (six-fold) uranium environments as found in $\mathrm{U}_{3} \mathrm{O}_{8}$ and in the $\mathrm{UO}_{3}$ polymorphs. Its occurrence in an eight-fold coordinated environment, however, is unique, and was hitherto only postulated by Conradson and coworkers in their EXAFS studies on $\mathrm{UO}_{2+x}$ and $\mathrm{PuO}_{2+x}{ }^{74,75} \mathrm{In}$ a recent examination of the local structure in $\mathrm{U}_{3} \mathrm{O}_{7}$ by EXAFS, the shortest U-O interatomic distances were modelled at 2.18(2) $\AA$, and displayed a mean thermal displacement value of $0.08(4) \AA .{ }^{38}$ Hence, bond lengths around $1.93 \AA$ were not resolved. The aim of the study was to evaluate the $\mathrm{U}_{3} \mathrm{O}_{7}$ trial model with cuboctahedral oxygen clusters and necessitated to define an average local environment consisting of three main classes of uranium-oxygen coordination. The refined structural model reported in the present work, however, contains up to 69 distinct bond lengths in the first coordination sphere of the different uranium sites. The short oxo-type bonds found in the $\mathrm{U} 1$ environment represent one out of 69 possibilities, thus contributing only marginally to the convoluted scattering profile at short $\mathrm{U}-\mathrm{O}$ distances. In addition, complementary oxygen shells could not be evaluated from the EXAFS data because the information limit was reached. ${ }^{38}$ The highest density of $\mathrm{U}-\mathrm{O}$ bonds occurs in the 2.1 to $2.5 \AA$ region (a histogram illustrating the axial distribution around each uranium site is available as Figure S3 in the ESI), which agrees well with the EXAFS evaluation.

Until now, the prevalent charge compensation mechanism for the $\mathrm{U}_{3} \mathrm{O}_{7}$ phase was embodied by a $1 / 3 \times U(I V)+2 / 3 U(V)$ distribution of uranium oxidation states. This was supported by High-Energy Resolution Fluorescence Detection (HERFD) X-ray Absorption Near-Edge Spectroscopy (XANES) at the U M4-edge that indicated a 36\% U(IV), and 64\% U(V) charge character in $\mathrm{U}_{3} \mathrm{O}_{7}{ }^{47}$ The electronic structure calculations and BVS assessment presented here 
similarly point towards a uranium charge distribution model involving predominantly U(IV) and $\mathrm{U}(\mathrm{V})$ states, but additionally, a small contribution of $\mathrm{U}(\mathrm{VI})$ states is highlighted. A closer look at the $\mathrm{U}_{60} \mathrm{O}_{140}$ structural model shows that some restrictions are imposed on the distribution of uranium chemical states based on site multiplicity rules. For example, the original scheme of $1 / 3 \times \mathrm{U}(\mathrm{IV})+2 / 3 \mathrm{U}(\mathrm{V})$ states, which corresponds to a $20 / 60 \times U(I V)+40 / 60 \mathrm{U}(\mathrm{V})$ distribution, can only be achieved if uranium site U1 (site multiplicity 4 , all other sites have a multiplicity of 8 ) is assigned a tetravalent state. Although this condition was assumed in the trial model, the atomic arrangements in the refined structure evolved towards a solution with a very compact U1 environment, which can only be associated to a higher valence state.

In this context it is interesting to compare the refined crystal structure of $\mathrm{U}_{3} \mathrm{O}_{7}$ to that of $\mathrm{Ca}_{2} \mathrm{YbF}_{7}(\mathrm{I} 4 / m)$, an ordered phase in the system $\mathrm{CaF}_{2}-\mathrm{YbF}_{3}$ which also exhibits a fluoriterelated superstructure. ${ }^{76,77}$ Their similarity is illustrated in the ESI (Figure S4), together with a graphic presentation of the orientation relation between both structures (Figure S5). The dominant crystallographic feature is the occurrence of cuboctahedral anion clusters in sheets perpendicular to the four-fold axis. Furthermore, within these sheets, the clusters are alternated by eight-fold coordinated (cubic) cation environments. In $\mathrm{Ca}_{2} \mathrm{YbF}_{7}$ the geometry of the cubic $\mathrm{CaF}_{8}$ sites is restrained by the cation valence state, i.e. $\mathrm{Ca}(\mathrm{II})$, and it remains regular. However, in uranium oxides such as $\mathrm{U}_{3} \mathrm{O}_{7}$, a wider variety of cation states can occur, enabling the equivalent $\mathrm{UO}_{8}$ site (U1) to become distorted in a much more compact environment. Most likely this mechanism is required to avoid steric hindering between the distorted and spatially more extended cuboctahedral oxygen clusters, as compared in $\mathrm{Ca}_{2} \mathrm{YbF}_{7}$.

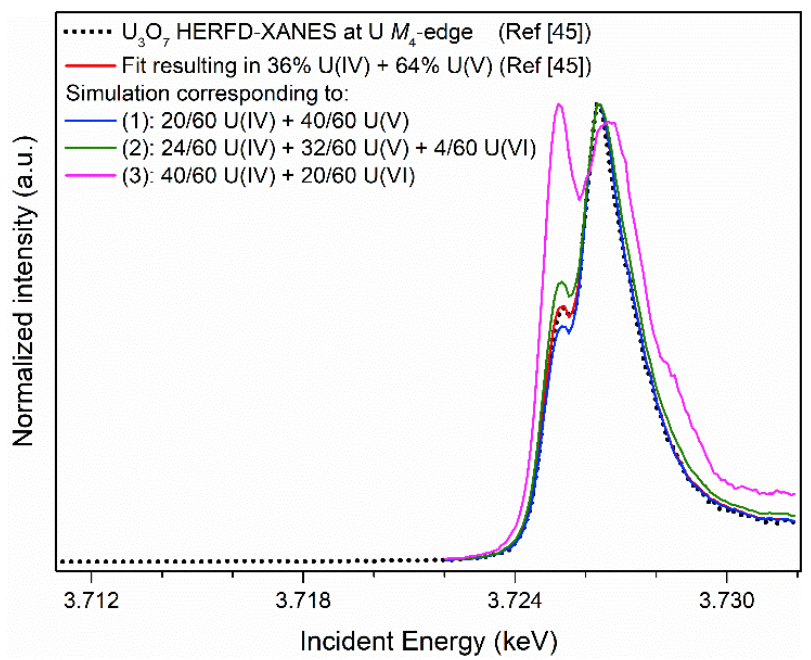

Figure 11. Experimental HERFD-XANES spectrum of $\mathrm{U}_{3} \mathrm{O}_{7}$ at the $\mathrm{U} M_{4}$-edge, reproduced from Leinders et al. ${ }^{47}$ Copyright 2017 American Chemical Society. The colored lines present fits to the spectrum obtained by convoluting spectra of the isolated tetra-, penta- and hexavalent components with the relative contributions indicated in the legend. While the third scheme (magenta line) can clearly be disregarded, a preference for either the first (blue line) or second scheme (green line) cannot be ascertained within the precision of the XANES measurement. 
The distribution of uranium oxidation states determined in this work introduces a new scheme to maintain charge neutrality in the $\mathrm{U}_{3} \mathrm{O}_{7}$ phase: $24 / 60 \times \mathrm{U}(\mathrm{IV})+32 / 60 \mathrm{U}(\mathrm{V})+4 / 60 \mathrm{U}(\mathrm{VI})$, which corresponds to a $40.0 \% \mathrm{U}(\mathrm{IV}), 53.3 \% \mathrm{U}(\mathrm{V})$, and $6.7 \% \mathrm{U}(\mathrm{VI})$ charge character. To compare the possible charge distribution models, the U M4-edge HERFD-XANES spectrum reported in Ref. 45 was simulated by convoluting spectra of the isolated tetra-, penta- and hexavalent components with the aforementioned relative contributions (see Figure 11). ${ }^{47} \mathrm{We}$ found that the experimental spectrum can be simulated with both the original and new scheme, leaving only a minor discrepancy for the intensity at the U(IV) shoulder. The local differences between experimental data and modelled spectra are of the same magnitude but inverse from each other, which shows that, within the precision of the XANES measurement, the unambiguous distinction between both charge distribution schemes cannot be made. It will be of specific interest to further investigate the evolution of hexavalent uranium states during progressive oxidation in the $\mathrm{U}-\mathrm{O}$ system, for instance, by comparing structural relations between the phases enveloping the $\mathrm{U}_{3} \mathrm{O}_{7}$ phase field, i.e. $\mathrm{U}_{4} \mathrm{O}_{9}$ and $\mathrm{U}_{3} \mathrm{O}_{8}$, respectively.

\subsection{Magnetic correlations predicted from first-principles calculations}

The electronic structure calculations evidence a strong interaction between $f$-electrons, producing a strong charge localization described as a charge-transfer insulating regime within the overall Mott-Hubbard mechanism. The low energy physics of insulator systems, in some cases, is described in terms of spin-only Hamiltonians: This happens for instance when the local symmetry of the $f$-element is low enough to lift the orbital degeneracy of the $f$-manifold. However, when the $f$-element possesses an orbital degeneracy in addition to that originating from spin, different kind of interactions become meaningful as for instance long-range correlations driven by Jahn-Teller, or exchange interactions that are responsible for orbital ordering and spin-gapped states.

The situation in $\mathrm{U}_{3} \mathrm{O}_{7}$ is possibly at the frontier between the two limit cases, because the structure retains some pseudosymmetries of the fluorite system, and additionally, a relatively strong relativistic spin-orbit coupling entangles locally spin and orbital degrees of freedom. The physics of such systems may differ from that of compounds in which SOC has a weaker perturbative nature, and it may produce magnetic interactions that depart from those described by the $\mathrm{SU}(2)$ symmetry. The strong charge localization occurring in the $\mathrm{U}_{3} \mathrm{O}_{7}$ structure, evidenced in the current work, introduces an interesting possibility because the triangular lattice of strongly magnetic U(IV) ions of the fluorite structure can be partially depleted by the presence of diamagnetic U(VI) ions, and the interactions can also be weakened by the existence of $U(V)$ ions. The introduction of SOC seems a suitable way of capturing the frustrated ferromagnetism in this insulating system because this coupling contribution can favor a spin canting of magnetic moments that would be otherwise aligned. 
The ab-initio model used for the electronic structure calculations detailed in section $\S 3.5$ started from the refined coordinates of the nuclear positions in the $\mathrm{P} 4_{2} / n$ space group and employed an automatic initialization of the Cartesian components of the magnetic moments of the uranium atoms. This particular choice converges to a possible magnetic structure solution in which the atomic charge distribution and the norm of the magnetic moments still respect the experimental tetragonal symmetry. However, the orientations of the magnetic moments produce a symmetry break that lifts the orbital degeneracy: The $\mathrm{P} 4_{2} / n$ symmetry of the nuclear structure is broken, as the 4-fold rotations are not maintained (see Table S6 in the ESI). The resulting magnetic structure is ferromagnetic and it can be described in the monoclinic subgroup P2'/c' of the tetragonal nuclear structure. To check the robustness of the initialization choice, we have performed a second calculation where the initialization of the magnetic moments obeys the requirement of this monoclinic subgroup. This calculation converges smoothly to a lower minimum, confirming the predictions of the preliminary calculation. A comparison of the uranium charges and amplitudes of the magnetic moments for the two initializations is available in the ESI (Figures S6 and S7).

The standard description of the magnetic subgroup $\mathrm{P} 2 \% c$ ' requires the following lattice transformation:

$$
\left(\mathbf{a}_{\mathbf{m}} \mathbf{b}_{\mathbf{m}} \mathbf{c}_{\mathbf{m}}\right)=(\mathbf{A} \quad \mathbf{B} \mathbf{C}) \cdot\left[\begin{array}{ccc}
-1 & 0 & 1 \\
0 & 0 & 1 \\
0 & 1 & 0
\end{array}\right]
$$

with $\mathbf{a}_{\mathbf{m}}, \mathbf{b}_{\mathbf{m}}, \mathbf{c}_{\mathbf{m}}$, and $\mathbf{A}, \mathbf{B}, \mathbf{C}$, the monoclinic and tetragonal basis vectors, respectively. The resulting monoclinic lattice parameters are equal to $a_{\mathrm{m}}=A=12.0018(3) \AA$, $b_{\mathrm{m}}=C=16.6239(7) \AA, c_{\mathrm{m}}=A \cdot \sqrt{2}=16.9731(4) \AA$, and $\beta=135^{\circ}$. The loss of symmetry elements of the paramagnetic group roughly doubles the number of independent atoms. The atomic positions of the 16 independent $\mathrm{U}$ atoms (and $42 \mathrm{O}$ atoms) are available as a magnetic CIF in the ESI, and additionally, the structural parameters of the magnetic uranium atoms are summarized in table S7. 


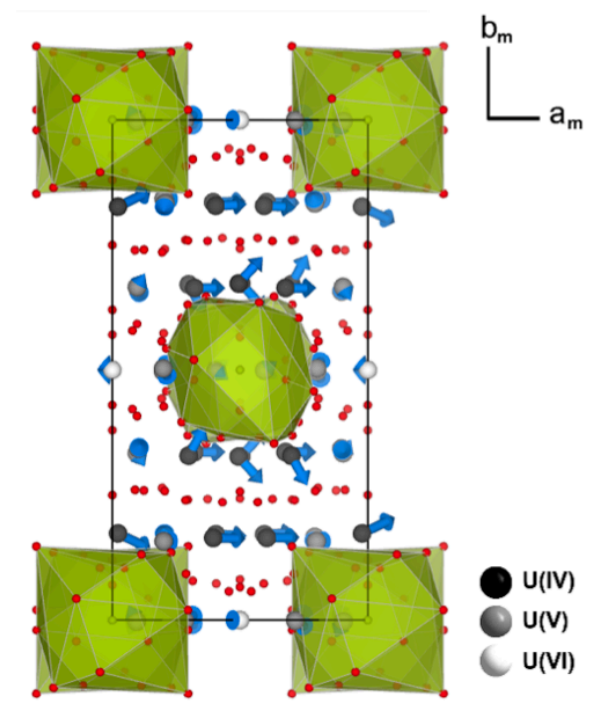

Figure 12. Representation of the $P 2^{\prime} / c^{\prime}$ magnetic structure, projected along the $\boldsymbol{c}_{\mathbf{m}}$-axis. The unit cell consists of stacked cation layers, 4 of them being independent because of the inversion symmetry. The bottom and middle layers contain the cuboctahedra centers and a mixture of $U(V)$ and $U(V I)$ atoms; the two intermediate layers contain a mixture of $U(I V)$ and $\mathrm{U}(\mathrm{V})$ and display the largest magnetic amplitudes.

A projection of the magnetic structure along the $\boldsymbol{c}_{\mathbf{m}}$ axis (see Figure 12) allows to distinguish a repeating stack of 4 cation layers having different characteristics. Each one of the independent $U$ atoms belongs to a particular layer so that the orientation relations of the magnetic moments among layers are not constrained by symmetry elements. The layer at the bottom of the stack $(\boldsymbol{a} 0 \boldsymbol{c})$ and the one at $(\boldsymbol{a} 1 / 2 \boldsymbol{c})$ contain the cuboctahedra centers, and localize the diamagnetic U(VI) ions (U1a, U1b) and two sets of U(V) ions (U3a, U3b, U5a, $\mathrm{U} 5 \mathrm{~b}$ ), see Figure 13. Half of these pentavalent atoms (U3b or U5a) carry magnetic moments roughly oriented along the $4 \boldsymbol{a}_{\mathbf{m}}-\boldsymbol{c}_{\mathbf{m}}$ direction while the other half (U3a or U5b) have their main component out of plane along the $\boldsymbol{b}_{\mathbf{m}}$ direction. Overall, these two layers display similar canted ferromagnetic arrangements. 

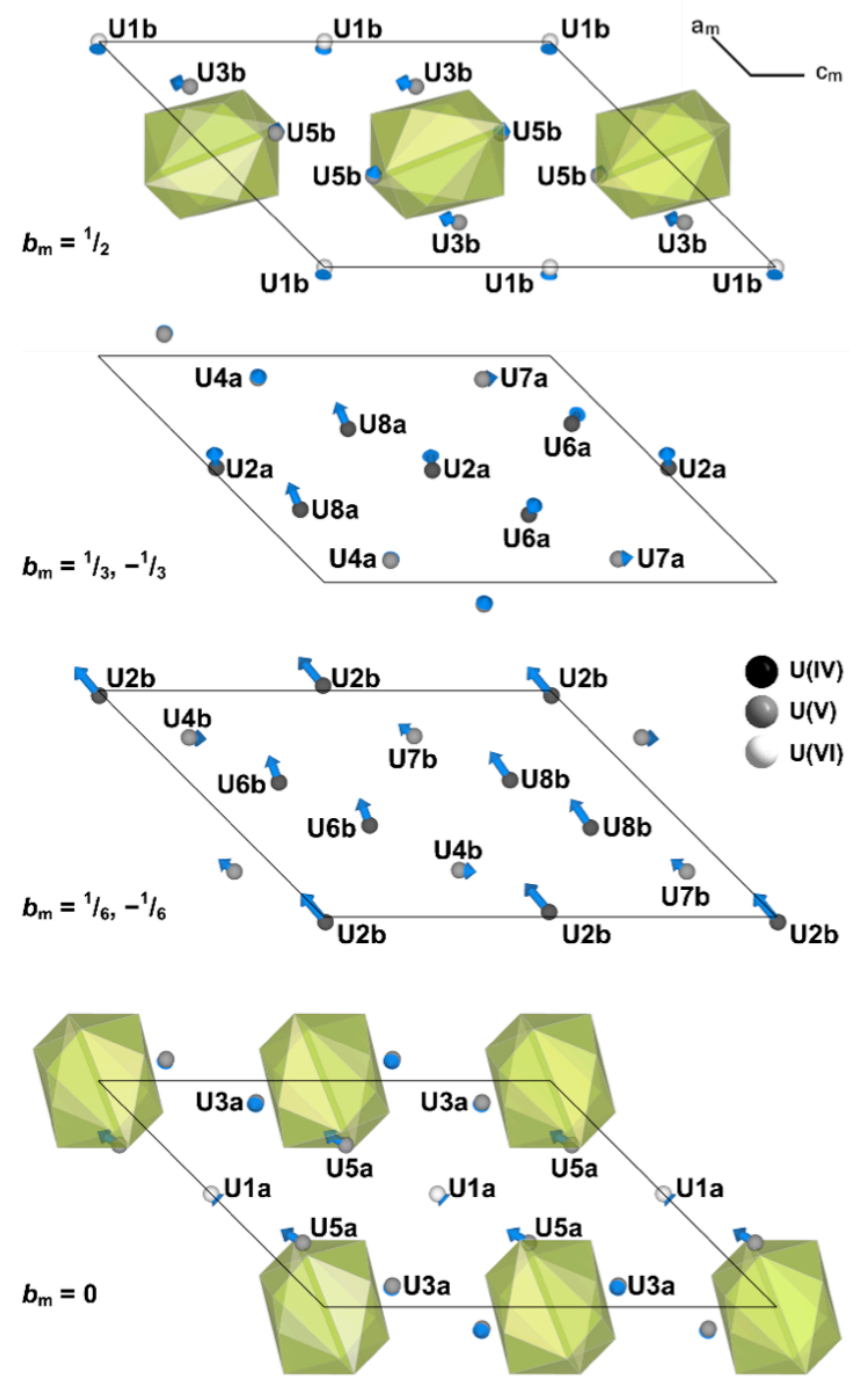

Figure 13. Projections of the P2'/c' magnetic structure along the $\boldsymbol{b}_{\mathbf{m}}$-axis, at elevations $b_{\mathrm{m}}$ corresponding to the four characteristic layers. To improve readability, the atomic radii were decreased by a factor 2, and all oxygen atoms were omitted. Within each layer a canted ferromagnetic order is observed: The main component of the amplitudes is roughly oriented along the $\boldsymbol{c}_{\mathrm{m}}$-direction in the bottom and middle layers, and along the $\boldsymbol{a}_{\mathbf{m}}$-direction in the intermediate layers.

The intermediate layers, corresponding to the planes $\left(\boldsymbol{a}^{1 / 6} \boldsymbol{c}\right)$ and $\left(\boldsymbol{a}^{1 / 3} \boldsymbol{c}\right)$, contain a mixture of $2 / 3 \mathrm{U}(\mathrm{IV})$ ions and $1 / 3 \mathrm{U}(\mathrm{V})$ ions but they have different magnetic arrangements in the two layers (see also Figure 13). In the second layer from the bottom, the three independent tetravalent ions (U2b, U6b, and U8b), with the largest magnetic moments, display a ferromagnetic order with the main component along the $\boldsymbol{a}_{\mathbf{m}}$ direction and a smaller out-ofplane component along $\boldsymbol{b}_{\mathbf{m}}$. The two independent pentavalent ions (U4b and U7b) have similar alignment, but half of them are flipped. In the third layer, the tetravalent ions display a variety of behaviors. One third of them (U8a) have an in-plane component that is still oriented roughly along $\boldsymbol{a}_{\mathbf{m}}$. Another third (U2a), all of those in capped square antiprisms adjacent to the oxygen cuboctahedra, have a large component along $\boldsymbol{b}_{\mathbf{m}}$ and a smaller in-plane component at $45^{\circ}$ with $\boldsymbol{a}_{\mathbf{m}}$. The last third (U6a) have also a large component along $\boldsymbol{b}_{\mathbf{m}}$ and an in-plane 
component oriented at about $90^{\circ}$ with $\boldsymbol{a}_{\mathbf{m}}$. Half of the pentavalent atoms in this layer (U4a) are aligned along $\boldsymbol{b}_{\mathbf{m}}$, the remaining ones (U7a) along $\boldsymbol{c}_{\mathbf{m}}$. In summary, while the nuclear arrangements are clearly fluorite-related, the ground state magnetic order in $\mathrm{U}_{3} \mathrm{O}_{7}$ displays a layered sub-structure.

We have used this structure to calculate the corresponding magnetic intensities in the neutron diffraction experiments (see Figure 14). The predicted magnetic intensities are very weak (the largest magnetic contribution to a reflection is only $0.2 \%$ of the main nuclear peak). These corrections to the nuclear intensities are comparable to the noise level of the scattering data that is $0.3 \%$ and $0.1 \%$ (relative intensity) for the D2B and D20 acquisitions, respectively. Therefore, while we are unable to further refine this magnetic model against the experimental data, the magnetic solution provided by the electronic structure minimization is correct within the accuracy of our experiment.

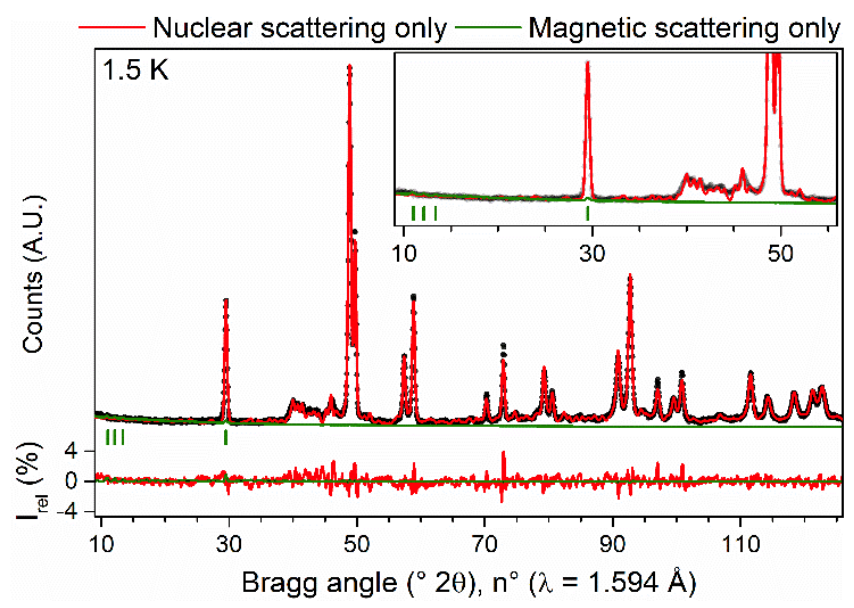

Figure 14. Comparison between the experimental neutron diffraction pattern acquired at $1.5 \mathrm{~K}$ at beamline D2B (black dots), and simulations assuming nuclear scattering (red line), or magnetic scattering only (green line). Vertical green lines indicate reflections from magnetic scattering with a relative intensity higher than $0.1 \%$. The contribution of the magnetic scattering is also reproduced on a relative intensity scale at the bottom of the graph, overlapped with the difference profile between experimental data and nuclear scattering fit. Clearly, the magnitude of the magnetic scattering is at the noise level of the diffraction data.

While the nuclear and magnetic structure models are fully consistent with the powder diffraction experiments, a more accurate assessment of the magnetic symmetry would require a polarized neutron scattering experiment on a single domain crystal that is not currently available for this compound. Alternatively, methods resulting in magnetic susceptibility or heat capacity data at cryogenic temperatures may be considered to further probe the magnetic properties. A further refinement of the electronic calculation would involve distinct Hubbard parameters for each one of the different environments of the uranium atoms and the eventual use of hybrid functionals. This might increase the current small dispersion in the magnetic moment amplitudes that we observe with a single Hubbard parameter, but this is not a trivial task considering that up to 16 distinct values can be expected. 


\section{Conclusion}

High-resolution and high-intensity neutron diffraction data were acquired at $1.5 \mathrm{~K}, 100 \mathrm{~K}$ and $298 \mathrm{~K}$ from a polycrystalline $\mathrm{U}_{3} \mathrm{O}_{7}$ powder sample prepared at thermal equilibrium. These data were comprehensively assessed to refine atomic arrangements in the crystallographic trial model proposed from prior investigations, i.e. the $\mathrm{U}_{60} \mathrm{O}_{140}$ superstructure model containing four cuboctahedral oxygen clusters. The refined structure, and specifically the anion sublattice, appears much more distorted than the trial model, confirming earlier observations which could not fully resolve the atomic arrangements in the $\mathrm{U}_{3} \mathrm{O}_{7}$ phase.

Three groups of uranium sites are distinguished, each associated to either a tetravalent (U2, U6, U8), pentavalent (U3, U4, U5, U7), or a hexavalent (U1) uranium state, leading to the charge-neutral valence state distribution $24 / 60 \times \mathrm{U}(\mathrm{IV})+32 / 60 \mathrm{U}(\mathrm{V})+4 / 60 \mathrm{U}(\mathrm{VI})$ in the $\mathrm{U}_{60} \mathrm{O}_{140}$ structural model at $1.5 \mathrm{~K}$. Uranium environments surrounding the distorted cuboctahedral oxygen clusters are nine- to ten-fold coordinated, and associate to the tetra- or pentavalent valence states. The centrosymmetric U1 environment is peculiar and consists of an eight-fold coordinated polyhedron (distorted cube) which contains two short $(2 \times 1.93(3) \AA$ ) oxo-type $\mathrm{U}-\mathrm{O}$ bonds, resulting in an overall very compact environment with a high local charge.

Self-consistent ab-initio calculations performed on the refined crystal structure provide novel insights on the electronic structure of $\mathrm{U}_{3} \mathrm{O}_{7}$. Firstly, we find that spin-orbit coupling is required to obtain semi-conductor properties. The valence and conduction bands are dominated by $\mathrm{O} 2 p$ and $\mathrm{U} 5 f$ states, respectively, suggesting that $\mathrm{U}_{3} \mathrm{O}_{7}$ can be described as a charge-transfer insulator, as the excitation takes place from the anion states, rather than among uranium states as in the Mott insulator picture characteristic of $\mathrm{UO}_{2}$.

Corroborating results with respect to the uranium speciation were further obtained from Bader and Hirshfeld-I charge analyses, establishing a very good correlation with the calculated magnetic moments carried by the uranium atoms, and also with bond-valence sum calculations. The occurrence of long-range antiferromagnetic order in $\mathrm{U}_{3} \mathrm{O}_{7}$ between $1.5 \mathrm{~K}$ and $100 \mathrm{~K}$ was ruled out by assessing the neutron diffraction data. The spin-resolved electronic structure calculations, on the other hand, predict a magnetic ground state structure in $\mathrm{U}_{3} \mathrm{O}_{7}$. Based on the orientation of the magnetic moments carried by the uranium atoms, a model was described in the $P 2 \% c$ ' magnetic space group. The magnetic structure displays canted ferromagnetic order in four characteristic layers, two consisting of mixed hexavalent (diamagnetic) and pentavalent $\left(0.8 \mu_{\mathrm{B}}\right)$ ions, and the other two consisting of tetravalent (1.6 $\left.\mu_{\mathrm{B}}\right)$ and pentavalent $\left(0.8 \mu_{\mathrm{B}}\right)$ ions. The predicted magnetic intensities are very weak and cannot be experimentally refined using powder diffraction data. To further probe the magnetic properties, dedicated experimental methods still need to be considered. 


\section{Acknowledgements}

Financial support for this research was partly provided by the Energy Transition Fund of the Belgian FPS Economy (Project SF-CORMOD - Spent Fuel CORrosion MODeling). This work was performed in part using HPC resources from GENCI-IDRIS (Grants 2020-101450 and 2020-101601), and in part by the VSC (Flemish Supercomputer Center) and the HPC infrastructure of the University of Antwerp (CalcUA), both funded by the FWO-Vlaanderen and the Flemish Government-department EWI. GL thanks E. Suard and C. Schreinemachers for assistance during the neutron scattering experiments at the ILL. GB acknowledges V. Petříček for suggestions on using JANA2006.

\section{Electronic supporting information}

Crystallographic Information Files (CIF) describing the refined structures at $1.5 \mathrm{~K}$ and $298 \mathrm{~K}$, and the predicted magnetic structure. Tables containing the full structural parameters of the crystallographic models referred to in the manuscript. Supporting results of the electronic structure calculations, and a histogram showing the variation in bond lengths around the uranium sites. 


\section{References}

(1) Fox, A. R., Bart, S. C., Meyer, K., Cummins, C. C., Towards uranium catalysts. Nature 2008, 455, 341-349.

(2) Ran, S., Eckberg, C., Ding, Q.-P., Furukawa, Y., Metz, T., Saha, S. R., Liu, I. L., Zic, M., Kim, H., Paglione, J., Butch, N. P., Nearly ferromagnetic spin-triplet superconductivity. Science 2019, 365, 684.

(3) Ao, B., Qiu, R., Tang, J., Chen, J., New theoretical insights into the actual oxidation states of uranium in the solid-state compounds. J. Nucl. Mater. 2021, 543, 152563.

(4) Szenknect, S., Alby, D., López García, M., Wang, C., Podor, R., Miserque, F., Mesbah, A., Duro, L., Zetterström Evins, L., Dacheux, N., Bruno, J., Ewing, R. C., Coffinite formation from UO2+x. Scientific Reports 2020, 10, 12168.

(5) Wiss, T., Benedetti, A., De Bona, E., 2.05 - Radiation Effects in $\mathrm{UO}_{2}$ in Comprehensive Nuclear Materials (Second Edition), Elsevier, Oxford, 2020, pp. 125148.

(6) Guéneau, C., Baichi, M., Labroche, D., Chatillon, C., Sundman, B., Thermodynamic assessment of the uranium-oxygen system. J. Nucl. Mater. 2002, 304, 161-175.

(7) Chevalier, P. Y., Fischer, E., Cheynet, B., Progress in the thermodynamic modelling of the O-U binary system. J. Nucl. Mater. 2002, 303, 1-28.

(8) Higgs, J. D., Lewis, B. J., Thompson, W. T., He, Z., A conceptual model for the fuel oxidation of defective fuel. J. Nucl. Mater. 2007, 366, 99-128.

(9) McEachern, R. J., Taylor, P., A review of the oxidation of uranium dioxide at temperatures below $400{ }^{\circ} \mathrm{C}$. J. Nucl. Mater. 1998, 254, 87-121.

(10) Wang, G., Batista, E. R., Yang, P., Water on Actinide Dioxide Surfaces: A Review of Recent Progress. Applied Sciences 2020, 10.

(11) Willis, B. T. M., Crystallographic studies of anion-excess uranium oxides. J. Chem. Soc., Faraday Trans. II 1987, 83, 1073-1081.

(12) Rousseau, G., Desgranges, L., Charlot, F., Millot, N., Nièpce, J. C., Pijolat, M., Valdivieso, F., Baldinozzi, G., Bérar, J. F., A detailed study of $\mathrm{UO}_{2}$ to $\mathrm{U}_{3} \mathrm{O}_{8}$ oxidation phases and the associated rate-limiting steps. J. Nucl. Mater. 2006, 355, 10-20.

(13) Desgranges, L., Baldinozzi, G., Rousseau, G., Nièpce, J.-C., Calvarin, G., Neutron diffraction study of the in situ oxidation of $\mathrm{UO}_{2}$. Inorg. Chem. 2009, 48, 7585-7592.

(14) Leinders, G., Pakarinen, J., Delville, R., Cardinaels, T., Binnemans, K., Verwerft, M., Low-temperature oxidation of fine $\mathrm{UO}_{2}$ powders: A process of nanosized domain development. Inorg. Chem. 2016, 55, 3915-3927.

(15) Leinders, G., Cardinaels, T., Binnemans, K., Verwerft, M., Low-Temperature Oxidation of Fine $\mathrm{UO}_{2}$ Powders: Thermochemistry and Kinetics. Inorg. Chem. 2018, 57, 4196-4204.

(16) Zaanen, J., Sawatzky, G. A., Allen, J. W., Band gaps and electronic structure of transition-metal compounds. Phys. Rev. Lett. 1985, 55, 418-421.

(17) Lander, G. H., Caciuffo, R., The fifty years it has taken to understand the dynamics of $\mathrm{UO}_{2}$ in its ordered state. J. Phys.: Condens. Matter 2020, 32, 374001.

(18) Dudarev, S. L., Liu, P., Andersson, D. A., Stanek, C. R., Ozaki, T., Franchini, C., Parametrization of LSDA $+U$ for noncollinear magnetic configurations: Multipolar magnetism in $\mathrm{UO}_{2}$. Physical Review Materials 2019, 3, 083802.

(19) Kindra, D. R., Evans, W. J., Magnetic Susceptibility of Uranium Complexes. Chem. Rev. 2014, 114, 8865-8882. 
(20) Amberger, H. D., Zur elektronenstruktur metallorganischer komplexe der f-elemente: $\mathrm{V}$. Interpretation der gegenwartig bekannten spektroskopischen und magnetochemischen eigenschaften von tris-(cyclopentadienyl)-uran(IV)-chlorid auf der grundlage der kristallfeld-theorie. J. Organomet. Chem. 1976, 116, 219-229.

(21) Anisimov, V. I., Zaanen, J., Andersen, O. K., Band theory and Mott insulators: Hubbard $U$ instead of Stoner I. Phys. Rev. B 1991, 44, 943-954.

(22) Perdew, J. P., Burke, K., Ernzerhof, M., Generalized Gradient Approximation Made Simple. Phys. Rev. Lett. 1996, 77, 3865-3868.

(23) Dudarev, S. L., Manh, D. N., Sutton, A. P., Effect of Mott-Hubbard correlations on the electronic structure and structural stability of uranium dioxide. Philos. Mag. B 1997, 75, 613-628.

(24) Dorado, B., Amadon, B., Freyss, M., Bertolus, M., DFT $+U$ calculations of the ground state and metastable states of uranium dioxide. Phys. Rev. B 2009, 79, 235125.

(25) He, H., Andersson, D. A., Allred, D. D., Rector, K. D., Determination of the Insulation Gap of Uranium Oxides by Spectroscopic Ellipsometry and Density Functional Theory. J. Phys. Chem. C 2013, 117, 16540-16551.

(26) Kvashnina, K. O., Kowalski, P. M., Butorin, S. M., Leinders, G., Pakarinen, J., Bès, R., Li, H., Verwerft, M., Trends in the valence band electronic structures of mixed uranium oxides. Chem. Commun. 2018, 54, 9757-9760.

(27) Pegg, J. T., Shields, A. E., Storr, M. T., Wills, A. S., Scanlon, D. O., de Leeuw, N. H., Magnetic structure of $\mathrm{UO}_{2}$ and $\mathrm{NpO}_{2}$ by first-principle methods. Phys. Chem. Chem. Phys. 2019, 21, 760-771.

(28) Andersson, D. A., Baldinozzi, G., Desgranges, L., Conradson, D. R., Conradson, S. D., Density functional theory calculations of $\mathrm{UO}_{2}$ oxidation: Evolution of $\mathrm{UO}_{2+x}$, $\mathrm{U}_{4} \mathrm{O}_{9-y}, \mathrm{U}_{3} \mathrm{O}_{7}$, and $\mathrm{U}_{3} \mathrm{O}_{8}$. Inorg. Chem. 2013, 52, 2769-2778.

(29) Wen, X.-D., Martin, R. L., Scuseria, G. E., Rudin, S. P., Batista, E. R., Burrell, A. K., Screened hybrid and DFT $+U$ studies of the structural, electronic, and optical properties of $\mathrm{U}_{3} \mathrm{O}_{8}$. J. Phys.: Condens. Matter 2012, 25, 025501.

(30) Brincat, N. A., Parker, S. C., Molinari, M., Allen, G. C., Storr, M. T., Density functional theory investigation of the layered uranium oxides $\mathrm{U}_{3} \mathrm{O}_{8}$ and $\mathrm{U}_{2} \mathrm{O}_{5}$. Dalton Transactions 2015, 44, 2613-2622.

(31) Vathonne, E., Wiktor, J., Freyss, M., Jomard, G., Bertolus, M., DFT $+U$ investigation of charged point defects and clusters in $\mathrm{UO}_{2}$. J. Phys.: Condens. Matter 2014, 26, 325501 .

(32) Andersson, D. A., Espinosa-Faller, F. J., Uberuaga, B. P., Conradson, S. D., Stability and migration of large oxygen clusters in $\mathrm{UO}_{2+\mathrm{x}}$ : Density functional theory calculations. J. Chem. Phys. 2012, 136, 234702.

(33) Brincat, N. A., Molinari, M., Parker, S. C., Allen, G. C., Storr, M. T., Computer simulation of defect clusters in $\mathrm{UO}_{2}$ and their dependence on composition. J. Nucl. Mater. 2015, 456, 329-333.

(34) Heyd, J., Scuseria, G. E., Ernzerhof, M., Hybrid functionals based on a screened Coulomb potential. The Journal of Chemical Physics 2003, 118, 8207-8215.

(35) Roy, L. E., Durakiewicz, T., Martin, R. L., Peralta, J. E., Scuseria, G. E., Olson, C. G., Joyce, J. J., Guziewicz, E., Dispersion in the Mott insulator $\mathrm{UO}_{2}$ : A comparison of photoemission spectroscopy and screened hybrid density functional theory. J. Comput. Chem. 2008, 29, 2288-2294.

(36) Wen, X.-D., Martin, R. L., Roy, L. E., Scuseria, G. E., Rudin, S. P., Batista, E. R., McCleskey, T. M., Scott, B. L., Bauer, E., Joyce, J. J., Durakiewicz, T., Effect of spinorbit coupling on the actinide dioxides $\mathrm{AnO}_{2}(\mathrm{An}=\mathrm{Th}, \mathrm{Pa}, \mathrm{U}, \mathrm{Np}, \mathrm{Pu}$, and $\mathrm{Am}): \mathrm{A}$ 
screened hybrid density functional study. The Journal of Chemical Physics 2012, 137, 154707.

(37) Tempest, P. A., Tucker, P. M., Tyler, J. W., Oxidation of uranium dioxide fuel pellets in air at 503 and $543 \mathrm{~K}$ studied using X-ray photoelectron spectroscopy and X-ray diffraction. J. Nucl. Mater. 1988, 151, 269-274.

(38) Leinders, G., Bes, R., Kvashnina, K. O., Verwerft, M., Local Structure in U(IV) and $\mathrm{U}(\mathrm{V})$ Environments: The Case of $\mathrm{U}_{3} \mathrm{O}_{7}$. Inorg. Chem. 2020, 59, 4576-4587.

(39) Leinders, G., Delville, R., Pakarinen, J., Cardinaels, T., Binnemans, K., Verwerft, M., Assessment of the $\mathrm{U}_{3} \mathrm{O}_{7}$ Crystal Structure by X-ray and Electron Diffraction. Inorg. Chem. 2016, 55, 9923-9936.

(40) Nowicki, L., Garrido, F., Turos, A., Thomé, L., Polytypic arrangements of cuboctahedral oxygen clusters in $\mathrm{U}_{3} \mathrm{O}_{7}$. J. Phys. Chem. Solids 2000, 61, 1789-1804.

(41) Li, Y., A fundamental understanding of the structures of oxygen defect clusters in $\mathrm{UO} 2+\mathrm{x}, \mathrm{U} 4 \mathrm{O} 9$ and U3O7: from the perspective of Tetris cubes. Acta Mater. 2020, 194, 482-495.

(42) Garrido, F., Ibberson, R. M., Nowicki, L., Willis, B. T. M., Cuboctahedral oxygen clusters in $\mathrm{U}_{3} \mathrm{O}_{7}$. J. Nucl. Mater. 2003, 322, 87-89.

(43) Blank, H., Ronchi, C., Electron diffraction of $\mathrm{U}_{4} \mathrm{O}_{9}$. Acta Crystallogr. A 1968, 24, 657-666.

(44) Bevan, D. J. M., Greis, O., Straehle, J., A new structural principle in anion-excess fluorite-related superlattices. Acta Crystallogr., Sect. A 1980, A36, 889-890, 882 plates.

(45) Bevan, D. J. M., Grey, I. E., Willis, B. T. M., The crystal structure of $\beta-U_{4} \mathrm{O}_{9-y} . J$. Solid State Chem. 1986, 61, 1-7.

(46) Kvashnina, K. O., Butorin, S. M., Martin, P., Glatzel, P., Chemical state of complex uranium oxides. Phys. Rev. Lett. 2013, 111, 253002.

(47) Leinders, G., Bes, R., Pakarinen, J., Kvashnina, K., Verwerft, M., Evolution of the Uranium Chemical State in Mixed-Valence Oxides. Inorg. Chem. 2017, 56, 67846787.

(48) Desgranges, L., Baldinozzi, G., Simeone, D., Fischer, H. E., Refinement of the $\alpha-\mathrm{U}_{4} \mathrm{O}_{9}$ Crystalline Structure: New Insight into the $\mathrm{U}_{4} \mathrm{O}_{9} \rightarrow \mathrm{U}_{3} \mathrm{O}_{8}$ Transformation. Inorg. Chem. 2011, 50, 6146-6151.

(49) Conradson, S. D., Andersson, D. A., Boland, K. S., Bradley, J. A., Byler, D. D., Durakiewicz, T., Gilbertson, S. M., Kozimor, S. A., Kvashnina, K. O., Nordlund, D., Rodriguez, G., Seidler, G. T., Bagus, P. S., Butorin, S. M., Conradson, D. R., Espinosa-Faller, F. J., Hess, N. J., Kas, J. J., Lezama-Pacheco, J. S., Martin, P., Martucci, M. B., Rehr, J. J., Valdez, J. A., Bishop, A. R., Baldinozzi, G., Clark, D. L., Tayal, A., Closure of the Mott gap and formation of a superthermal metal in the Fröhlich-type nonequilibrium polaron Bose-Einstein condensate in $\mathrm{UO}_{2+x}$. Phys. Rev. $B$ 2017, 96, 125114.

(50) Deutsch, M., Förster, E., Hölzer, G., Härtwig, J., Hämäläinen, K., Kao, C. C., Huotari, S., Diamant, R., X-ray spectrometry of copper: New results on an old subject. J. Res. Natl. Inst. Stand. Technol. 2004, 109, 75-98.

(51) Leinders, G., Baldinozzi, G., Lamoen, D., Ritter, C., Schreinemachers, C., Suard, E., Verwerft, M., The crystal structure of U3O7: Refinement of oxygen positions from neutron diffraction data. 2019. Institut Laue-Langevin (ILL) doi:10.5291/ILLDATA.5-21-1119

(52) Leinders, G., Ritter, C., Investigating the magnetic ordering at low temperatures in U3O7. 2019. Institut Laue-Langevin (ILL) doi:10.5291/ILL-DATA.EASY-493 
(53) Petříček, V., Dušek, M., Palatinus, L., in Zeitschrift für Kristallographie - Crystalline Materials, Vol. 229, 2014, p. 345.

(54) Kresse, G., Furthmüller, J., Efficient iterative schemes for ab initio total-energy calculations using a plane-wave basis set. Phys. Rev. B 1996, 54, 11169-11186.

(55) Blöchl, P. E., Projector augmented-wave method. Phys. Rev. B 1994, 50, 17953 17979.

(56) Kresse, G., Joubert, D., From ultrasoft pseudopotentials to the projector augmentedwave method. Phys. Rev. B 1999, 59, 1758-1775.

(57) Kotani, A., Yamazaki, T., Systematic Analysis of Core Photoemission Spectra for Actinide Di-Oxides and Rare-Earth Sesqui-Oxides. Progress of Theoretical Physics Supplement 1992, 108, 117-131.

(58) Meredig, B., Thompson, A., Hansen, H. A., Wolverton, C., van de Walle, A., Method for locating low-energy solutions within DFT+U. Phys. Rev. B 2010, 82, 195128.

(59) Blöchl, P. E., Jepsen, O., Andersen, O. K., Improved tetrahedron method for Brillouinzone integrations. Phys. Rev. B 1994, 49, 16223-16233.

(60) Setyawan, W., Curtarolo, S., High-throughput electronic band structure calculations: Challenges and tools. Computational Materials Science 2010, 49, 299-312.

(61) Henkelman, G., Arnaldsson, A., Jónsson, H., A fast and robust algorithm for Bader decomposition of charge density. Computational Materials Science 2006, 36, 354-360.

(62) Bultinck, P., Van Alsenoy, C., Ayers, P. W., Carbó-Dorca, R., Critical analysis and extension of the Hirshfeld atoms in molecules. The Journal of Chemical Physics 2007, $126,144111$.

(63) Westrum, E. F., Jr., Gronvold, F., Triuranium heptaoxides: heat capacities and thermodynamic properties of $\alpha$ - and $\beta-\mathrm{U}_{3} \mathrm{O}_{7}$ from 5 to 350 K. Phys. Chem. Solids 1962, 23, 39-53.

(64) Taylor, P., Burgess, E. A., Owen, D. G., An X-ray diffraction study of the formation of beta-uranium oxide $\left(\mathrm{UO}_{2.33}\right)$ on uranium dioxide pellet surfaces in air at 229 to 275 ${ }^{\circ}$ C. J. Nucl. Mater. 1980, 88, 153-160.

(65) Frazer, B. C., Shirane, G., Cox, D. E., Olsen, C. E., Neutron-Diffraction Study of Antiferromagnetism in $\mathrm{UO}_{2}$. Physical Review 1965, 140, A1448-A1452.

(66) Huntzicker, J. J., Westrum, E. F., The magnetic transition, heat capacity, and thermodynamic properties of uranium dioxide from 5 to $350 \mathrm{~K}$. The Journal of Chemical Thermodynamics 1971, 3, 61-76.

(67) Willis, B. T. M., Structures of $\mathrm{UO}_{2}, \mathrm{UO}_{2+x}$ and $\mathrm{U}_{4} \mathrm{O}_{9}$ by neutron diffraction. J. Phys. (Paris) 1964, 25, 431-439.

(68) Murray, A. D., Willis, B. T. M., A neutron diffraction study of anion clusters in nonstoichiometric uranium dioxide. J. Solid State Chem. 1990, 84, 52-57.

(69) Jones, D. J., Roziere, J., Allen, G. C., Tempest, P. A., The structural determination of fluorite- type oxygen excess uranium oxides using EXAFS spectroscopy. The Journal of Chemical Physics 1986, 84, 6075-6082.

(70) Willardson, R. K., Moody, J. W., Goering, H. L., The electrical properties of uranium oxides. J. Inorg. Nucl. Chem. 1958, 6, 19-33.

(71) Krupa, J. C., Carnall, W. T., Electronic structure of U4+, Np4+, and Pu4+ doped into ThSiO4 single crystal. The Journal of Chemical Physics 1993, 99, 8577-8584.

(72) Bader, R. F. W., The Quantum Mechanical Basis of Conceptual Chemistry. Monatshefte für Chemie / Chemical Monthly 2005, 136, 819-854.

(73) Zachariasen, W. H., Bond lengths in oxygen and halogen compounds of $\mathrm{d}$ and $\mathrm{f}$ elements. J. Less Common Met. 1978, 62, 1-7. 
(74) Conradson, S. D., Manara, D., Wastin, F., Clark, D. L., Lander, G. H., Morales, L. A., Rebizant, J., Rondinella, V. V., Local Structure and Charge Distribution in the UO2U4O9 System. Inorg. Chem. 2004, 43, 6922-6935.

(75) Conradson, S. D., Begg, B. D., Clark, D. L., den Auwer, C., Ding, M., Dorhout, P. K., Espinosa-Faller, F. J., Gordon, P. L., Haire, R. G., Hess, N. J., Hess, R. F., Webster Keogh, D., Lander, G. H., Manara, D., Morales, L. A., Neu, M. P., Paviet-Hartmann, P., Rebizant, J., Rondinella, V. V., Runde, W., Drew Tait, C., Kirk Veirs, D., Villella, P. M., Wastin, F., Charge distribution and local structure and speciation in the $\mathrm{UO}_{2+x}$ and $\mathrm{PuO}_{2+x}$ binary oxides for $x \leqslant 0.25$. J. Solid State Chem. 2005, 178, 521-535.

(76) Ness, S. E., Bevan, D. J. M., Rossell, H. J., Cuboctahedral Anion Clusters in FluoriteRelated Superstructures: The Structure of $\mathrm{Ca}_{2} \mathrm{YbF}_{7}$. Part 1. Eur. J. Solid State Inorg. Chem. 1988, 25, 509-516.

(77) Bevan, D. J. M., McCall, M. J., Ness, S. E., Taylor, M. R., Cuboctahedral Anion Clusters in Fluorite-Related Superstructures: The Structure of $\mathrm{Ca}_{2} \mathrm{YbF}_{7}$. Part 2. Eur. J. Solid State Inorg. Chem. 1988, 25, 517-526. 


\section{TOC graphic}

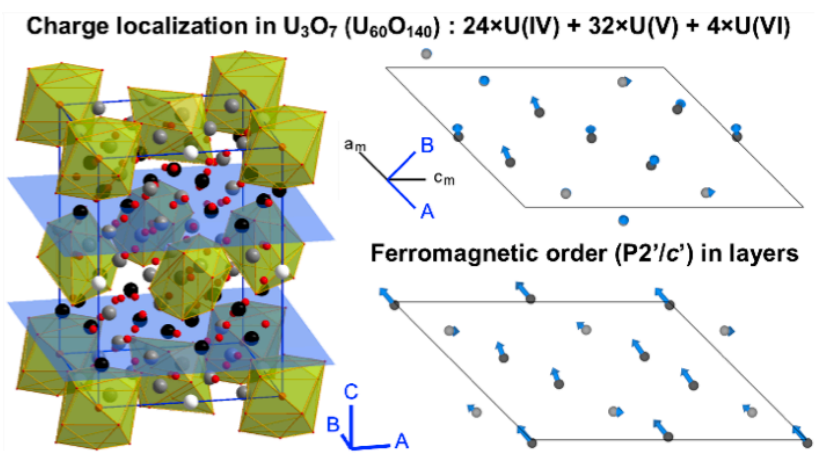

\section{Synopsis}

Atomic arrangements in the mixed-valence oxide $\mathrm{U}_{3} \mathrm{O}_{7}$ are refined from neutron scattering data. By combining experiment with electronic structure calculations, an interplay between uranium charge localization and the magnetic moments carried by the uranium atoms is revealed. Most cations have tetravalent and pentavalent states, and carry magnetic moments of respectively $1.6 \mu_{\mathrm{B}}$ and $0.8 \mu_{\mathrm{B}}$. One particularly compact environment is assigned a hexavalent state, resulting in a $24 / 60 \times \mathrm{U}(\mathrm{IV})+32 / 60 \mathrm{U}(\mathrm{V})+4 / 60 \mathrm{U}(\mathrm{VI})$ charge distribution. 